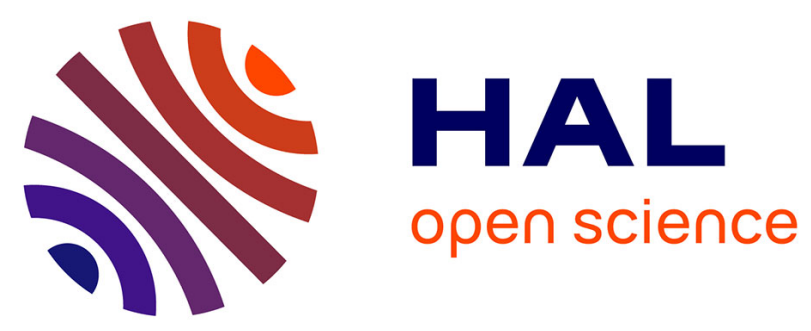

\title{
Numerical analysis of truncation error, consistency, and axis boundary condition for axis-symmetric flow simulations via the radius weighted lattice Boltzmann model
}

Damien David, Frederic Kuznik, Kevyn Johannes, Lucie Merlier

\section{To cite this version:}

Damien David, Frederic Kuznik, Kevyn Johannes, Lucie Merlier. Numerical analysis of truncation error, consistency, and axis boundary condition for axis-symmetric flow simulations via the radius weighted lattice Boltzmann model. Computers and Fluids, 2015, 116, pp.46-59. 10.1016/j.compfluid.2015.04.010 . hal-01287488

\section{HAL Id: hal-01287488 \\ https://hal.science/hal-01287488}

Submitted on 13 Mar 2016

HAL is a multi-disciplinary open access archive for the deposit and dissemination of scientific research documents, whether they are published or not. The documents may come from teaching and research institutions in France or abroad, or from public or private research centers.
L'archive ouverte pluridisciplinaire HAL, est destinée au dépôt et à la diffusion de documents scientifiques de niveau recherche, publiés ou non, émanant des établissements d'enseignement et de recherche français ou étrangers, des laboratoires publics ou privés. 


\title{
Numerical analysis of truncation error, consistency, and axis boundary condition for axis-symmetric flow simulations via the radius weighted lattice Boltzmann model
}

\author{
Damien DAVID ${ }^{\mathrm{a}, \mathrm{b}, *}$, Frederic Kuznik ${ }^{\mathrm{a}, \mathrm{c}}$, Kevyn Johannes ${ }^{\mathrm{a}, \mathrm{b}}$, Lucie Merlier ${ }^{\mathrm{a}, \mathrm{c}}$ \\ ${ }^{a}$ CETHIL, UMR5008, 9 rue de la physique, 69621 Villeurbanne, France \\ ${ }^{b}$ Universite Claude Bernard Lyon 1 \\ ${ }^{c}$ INSA de Lyon
}

\begin{abstract}
This paper is the continuity of Guo's work [Phys.Rev., 2009, 046708] about a lattice Boltzmann model for axis-symmetric flows. This is a radius weighted LBM model: all the moments are proportional to the radial coordinate $r$. A Taylor series analysis is performed on the discrete Boltzmann Model in order to assess the consistency. The truncation error terms do not indicate any error increase along the radial direction, but they contain a spurious term $u_{r} / r$ which considerably reduces the model accuracy when radial velocity is non-null: simulation results highlight a reductions of the order of accuracy (from second to first). A new axis boundary condition was created to improve the model accuracy and to cope with the spurious error term. It was assessed though several test cases.
\end{abstract}

Keywords: Lattice Boltzmann models, Axis-symmetric flows, Truncation Error Analysis, Boundary conditions

\footnotetext{
*Corresponding author: damien.david@insa-lyon.fr
} 


\section{Nomenclature}

$\begin{array}{ll}\vec{a} & \text { acceleration } \\ \overrightarrow{c_{k}} & \text { particle speeds } \\ c_{s}=1 / 3 & \text { sound speed } \\ p & \text { pressure } \\ f_{k} & \text { distribution functions } \\ R_{k} & \text { force terms in LB equation } \\ r & \text { radius coordinate } \\ \vec{u} & \text { speeds }\end{array}$

Greek symbols

$\begin{array}{ll}\nu & \text { kinetic viscosity } \\ \rho & \text { density } \\ \tau & \text { relaxation parameter } \\ \Delta t & \text { time step } \\ \Delta X & \text { space step } \\ \text { Subscripts } & \\ i, j & \text { node index } \\ k & \text { particle velocity index } \\ t & \text { Time } \\ \alpha, \beta \gamma & \text { Einstein notation indexes } \\ r, z & \text { radial / vertical direction }\end{array}$

\section{Introduction}

The lattice Boltzmann method (LB method) is a well understood method for the simulation of fluid flows. Although it is limited to second order accuracy, it can compete with traditional fluid simulation methods in terms of computational performances, and simplicity of the implementation.

Historical origins of the method are found in the kinetic theory: as a continuity of Lattice Gas Cellular Automata (Frisch et al.[1], McNamara and Zanetti [2]), or as a special discretization of the Boltzmann equation (He and Luo [3]). 
The use of lattice Boltzmann models is not restricted to the simulation of the microscopic behaviour of fluids. The method is also a powerful numerical tool for the simulation of partial differential equations. Thus, LB models exist for various differential equations: Full Navier Stokes equations (Qian et al. [4]), Poisson equation (Chai and Shi [5]), Fourier equation (Mishra [6]), etc... For each LBM model, it is essential that the consistency is expressed at least in one journal paper. Moreover, a truncation error analysis might be helpful for the determination of the optimal model parameters, and for the interpretation of the model behaviour under certain circumstances.

The consistency is assessed when the numerical equations tends toward the appropriate partial differential equations as the discretization parameters $\Delta X$ and $\Delta t$ decrease. The consistency of LB models is traditionally established through the Chapman Enskog expansion. This method is an heritage from the kinetic theory: it involves a scale separation (between acoustic and diffusive transport) on the distribution functions and the differential operators. Although the method is commonly used for recovering the zero order terms of the simulated equations, it might become a very long and painful experience to use it for recovering the truncation error terms unless restricted scaling hypothesis are adopted (Zhao [7]).

An alternative method was proposed by Holdych [8]: the so-called "Taylor series analysis". The method is an extension of Junk's work [9] for $\tau=1$ to any value of the relaxation parameter. It does not involve any scale separation that might be confusing for LBM neophytes. It offers a clear an simple technique to get to the zero order equations, and does not require much more effort to reach the whole expression of truncation error terms. Despite its inherent simplicity, the method is still not popular among the LBM community (Ginzburg [10]).

The present work was motivated by the need of a precise and reliable axissymmetric flow model, to simulate low-Rayleigh flows inside some cylindrical capsules that contain phase change materials. The model which was selected is the model proposed by Guo [11]. Most of the axis-symmetric LB models were obtained by modifying existing Cartesian LB models in order to recover the 
additional terms in cylindrical Navier-Stokes equations. Halliday et al. [12], Reis and Philipps [13, 14], and Chen et al. [15, 16, 17, 18] used virtual source terms. Zhou $[19,20]$ used a radius-dependent relaxation time and a virtual source term.

The way Guo built his model is radically different. The author started from the continuous Boltzmann equation in cylindrical coordinates from Bergers [21]. He discretized the equations and obtained a new kind of LB model, where the radius coordinate appears in all the moments definitions. From now, we refer to this model as the Radius-Weighted Lattice Boltzmann model: RW-LB model.

Despite subsequent extensions of Guo's model to thermal flows (Zheng et al. [22]), and multi-relaxation time collision operator (Wang et al. [23]), the consistency of the discrete model has never been clearly assessed. Guo [11] applied the Chapman Enskog expansion to the continuous Boltzmann equations from which he built the discrete RW-LB model. Moreover, the presence of the radial coordinate in the moments definitions and the force term might modify the truncation error terms: its effect on the model precision must be clearly stated. This is the first purpose of the present paper, which is treated through a Taylor series analysis.

The second purpose of the present paper concerns the boundary condition near the symmetry axis. Guo proposed a "mirror" boundary condition. This boundary condition is acceptable for simulating a symmetry plane in Cartesian coordinates. It is not necessarily suited for the RW-LB model. A new and more appropriate boundary condition is defined in the subsequent sections.

The present paper is organized as follow. The Navier-Stokes equations for axis-symmetric flows and the RW-LB model are quickly reminded in the first two sections. Then, the consistency of the model is tested and the error terms are expressed from the Taylor series analysis. An alternative axis boundary condition is proposed in the fourth section. Finally, the model is tested with the new boundary condition on several test cases. 


\section{Macroscopic equations}

Cylindrical formulations of the Navier-Stokes equations are found in every fluid dynamics textbook. The following formulation facilitates the comparison with the macroscopic equations obtained through the Taylor series analysis.

$$
\begin{aligned}
& \partial_{\beta}\left(r u_{\beta}\right)=0 \\
& \begin{aligned}
\partial_{t}\left(r u_{\alpha}\right)+\partial_{\alpha \beta}\left(r u_{\alpha} u_{\beta}\right)=- & \frac{1}{\rho} \partial_{\alpha}(r p)+\frac{p}{\rho} \delta_{\alpha r} \ldots \\
& \cdots+\nu\left[\partial_{\beta \beta}\left(r u_{\alpha}\right)-\partial_{r}\left(u_{\alpha}\right)-\frac{u_{\alpha}}{r} \delta_{\alpha r}\right]+\frac{r F_{0 \alpha}}{\rho}
\end{aligned}
\end{aligned}
$$

In the present paper, we adopt the Einstein formulation for all the equations. In equations 1 and 2 , the summation index is $\beta$ and $\alpha$ is a free index.

\section{RW-LB model}

The RW-LB model is a D2Q9 BGK model, where the moments and the equilibrium distribution functions are multiples of the radial coordinate $r$. The simulation domain is discretized into square grids. The grid size is $\Delta X$. The time step is $\Delta t$. Each grid point is connected with itself and the eight neighbouring nodes through nine particle velocities:

$$
\begin{aligned}
& \vec{c}_{0}=(0 ; 0) \Delta X / \Delta t \\
& \vec{c}_{1-4}=(0 ; \pm 1) \Delta X / \Delta t, \quad( \pm 1,0) \Delta X / \Delta t \\
& \vec{c}_{5-9}=( \pm 1 ; \pm 1) \Delta X / \Delta t
\end{aligned}
$$

The distribution functions $f_{0-8}$ follow the governing equation:

$$
f_{k}\left(\vec{x}+\vec{c}_{k} \Delta t, t+\Delta t\right)-f_{k}(\vec{x}, t)=-\frac{1}{\tau}\left(f_{k}-f_{k}^{e q}\right)+\Delta t\left(1-\frac{1}{2 \tau}\right) R_{k}
$$

The discrete relaxation time $\tau$ is related to the viscosity of the fluid through $\nu=c_{s}^{2} \Delta t(\tau-0,5)$, where $c_{s}^{2}=\Delta X^{2} / \sqrt{3} \Delta t^{2}$ is the sound speed. 
$f_{k}^{e q}$ is the discretized Maxwellian equilibrium distribution function multiplied by $r$. It is given by Equation 5, where $\omega_{k}$ is a weighting which depends on the associated particle velocity: $\omega_{0}=4 / 9, \omega_{1-4}=1 / 9, \omega_{5-9}=1 / 36$.

$$
f_{k}^{e q}=\rho r \omega_{k}\left[1+\frac{\overrightarrow{c_{k}} \cdot \vec{u}}{c_{s}^{2}}+\frac{\left(\overrightarrow{c_{k}} \cdot \vec{u}\right)^{2}}{2 \cdot c_{s}^{4}}-\frac{\vec{u}^{2}}{2 \cdot c_{s}^{2}}\right]
$$

The distribution functions $f_{k}$ are related to the macroscopic variables through the first and second order moments:

$$
\begin{aligned}
& \sum_{k} f_{k}=r \rho \\
& \sum_{k} c_{k \alpha} \cdot f_{k}=r\left(\rho u_{\alpha}-\Delta t \frac{F_{\alpha}}{2}\right)
\end{aligned}
$$

For the source term $R_{k}$, two formulations are available. The first formulation (Equation 8) is the He-type formulation (He et al. [24]) proposed by Guo [11] for the original RW-LB model. The second formulation (Equation 9) is the Guo-type formulation from Guo et al. [25]. Since both formulations have the same zero-th, first, and second order moments, they lead to the same macroscopic equations and second order truncation error terms. Both formulations are equivalent for this study. The second formulation is chosen to get lighter expressions.

$$
\begin{array}{ll}
R_{k}^{\mathrm{He}}=\frac{\left(\overrightarrow{c_{k}}-\vec{u}\right) \cdot \vec{F}}{\rho r c_{s}^{2}} f_{k}^{e q} & \text { He-type } \\
R_{k}=\omega_{k}\left[\frac{\overrightarrow{c_{k}}-\vec{u}}{c_{s}^{2}}+\frac{\overrightarrow{c_{k}} \cdot \vec{u}}{c_{s}^{4}} \overrightarrow{c_{k}}\right] \cdot(r \vec{F}) & \text { Guo-type }
\end{array}
$$

The force $F_{\alpha}$ is the sum of the "real" force applied to the fluid $F_{0 \alpha}$, and a corrective pseudo-force required to recover the cylindrical Navier Stokes equations $F_{\alpha}^{\prime}$ :

$$
F_{\alpha}=F_{0 \alpha}+F_{\alpha}^{\prime}=F_{0 \alpha}+\left(\frac{\rho c_{s}^{2}}{r}-\rho \nu \frac{2 u_{r}}{r^{2}}\right) \delta_{\alpha r}
$$


Please note that Equation 10 is an exact reformulation of the force term given by Guo. The formulation given by Wang et al. [23] and Zheng et al. [22] is wrong: they confused the continuous model relaxation time $\tau^{*}=\nu / c_{s}^{2}$ with the discrete model relaxation time $\tau=\tau^{*} / \Delta t+0.5$

\section{Consistency and truncation error}

\subsection{Scaling strategy}

In LB models, the quantities $\nu, \tau, \Delta X$ and $\Delta t$ are connected through the relation:

$$
\nu=\frac{\Delta X^{2}}{3 \Delta t}\left(\frac{2 \tau-1}{2}\right)
$$

Because of this relationship, it is impossible to modify $\Delta X$ (or $\Delta t$ ) without modifying other parameters of the model. Thus, whenever a study involves mesh refinement of a LBM model (error analysis, consistency, local mesh refinement...), it is important to clarify the condition of the mesh refinement: which parameter remains constant? How does $\Delta t$ vary with $\Delta X$ ?

The choice of the constant parameter concerns two reasonable options: either the relaxation time $\tau$ is constant, or the Mach number $M a$ is constant. Both parameters participate to the stability and the accuracy of the model. It is now common knowledge that acceptable values of $\tau$ lies in a narrow range between 0.5 and 2, and that the Mach number should be as low as possible to avoid compressibility effects.

The literature proposes two common scaling options. Let $\Delta X=1 / N_{X}$ be the reference small parameter. Acoustic scaling involves $\Delta t \propto \Delta X$, and diffusive scaling involves $\Delta t \propto \Delta X^{2}$ (Asinari et al. [26], Junk [9]).

The different mesh refinement strategies were evaluated by computing the following quantities: reference velocity $U_{0}$, model viscosity $\nu$, Mach number $M a$, relaxation parameter $\tau$ and number of time steps $N_{t}$. Those calculation were based on a fixed Reynolds number $R e$ and non dimension simulated time $\kappa$ : 


$$
M a=\frac{U_{0}}{\Delta X /(\sqrt{3} \Delta t)} \quad R e=\frac{U_{0} N_{X} \Delta X}{\nu} \quad \kappa=\frac{U_{0} N_{t} \Delta t}{N_{X} \Delta X}
$$

When the Mach number is fixed, we get $\tau-0,5 \propto N_{X}$. We might reach unreasonably high values of $\tau$ by refining the mesh. On the contrary, by fixing $\tau$, we get $M a \propto 1 / N_{X}$ : we reduce the Mach number value by refining the mesh. That's why we choose to fix the value of $\tau$ during the mesh refinement.

Since the aim of the paper is not to compare the relative order of convergence obtained through either acoustic or diffusive scaling, there was no such a strict criterion for choosing between the scaling strategies. None of them leads to a faster convergence, we always get

$$
N_{t}=\frac{\kappa N_{X}^{2}}{\operatorname{Re}(\tau-0.5)}
$$

We chose diffusive scaling based on recommendations from Holdych et al. [8]. With this scaling strategy, the velocity does not depend on $N_{X}$, and the viscosity can be adjusted through the ratio $\Delta X^{2} / \Delta t$ (Equation 11).

\subsection{Taylor series analysis}

A rigorous application of the Taylor series analysis to the RW-LB model is detailed in appendix Appendix A. Here, we present the major results of the calculations.

The Taylor series analysis leads to the modified equations of the numerical scheme. For the continuity equation, we get:

$$
\partial_{\alpha}\left(r u_{\alpha}\right)=\frac{(2 \tau-1)^{2}}{12 \nu} \Delta X^{2}\left[-\frac{r}{\rho \nu}\left(\partial_{t} p+u_{\alpha} \partial_{\alpha} p\right)+\nu \partial_{r}\left(\frac{u_{r}}{r}\right)\right]+O\left(\Delta X^{4}\right)
$$

and for the momentum equation, we get: 


$$
\begin{aligned}
& 0=-\partial_{t}\left(r u_{\alpha}\right)-\partial_{\beta}\left(r u_{\alpha} u_{\beta}\right)-\frac{\partial_{\alpha}(r p)}{\rho}+\frac{p}{\rho} \delta_{\alpha r} \ldots \\
& \cdots+\nu\left[\partial_{\beta \beta}\left(r u_{\alpha}\right)-\partial_{r}\left(u_{\alpha}\right)-\delta_{\alpha r}\left(\frac{u_{r}}{r}\right)\right]+r \frac{F_{0 \alpha}}{\rho}+\Delta X^{2} M E+O\left(\Delta X^{4}\right)
\end{aligned}
$$

where the momentum truncation error $M E$ is expressed in Equation A.37 of appendix Appendix A.

The zero order elements of Equations 14 and 15 are identical to the cylindrical Navier Stokes Equations 1 and 2. The consistency of the model is assessed wherever the truncation error do not reach infinite values.

One concern that might arise with the RW-LB model is the repartition of the error along the radial coordinate, due to the presence of $r$ in the definition of the moments. The expression of the error terms can give us some answers about this concern. There are two kinds of error terms in Equations 14 and A.37. The first ones are functions of $r p$ or $r \vec{u}$. The other ones are functions of $u_{r} / r$.

The first kind of error terms are functions of quantities that are proportional to $r$. However, Equations 14 and 15 govern the evolution of $r \rho$ and $r \rho \vec{u}$, which are also proportional to $r$. The $r$-proportionality in the error and the main equation terms compensate each other. The first kind of error terms should not involve any increasing trend along the radius coordinate.

If the radial velocity $u_{r}$ is null, the error terms depending on $u_{r} / r$ are null and there is no singularity near the axis. However, with non-zero radial velocities, the term $u_{r} / r$ reaches extreme values near $r=0$, the error terms can not be neglected along the symmetry axis. For that reason, we conclude that the consistency of the model is assessed with no restriction if $u_{r}$ is null; it is assessed in all the model except at the axis location when $u_{r}$ is not null. This restriction is not limiting: axis-symmetric simulations are performed on a half-section of the cylindrical domain, and the symmetry axis is treated with proper boundary conditions. The accuracy of the model near the axis depends on the quality of 
the boundary condition.

Finally, let's consider the evolution of the total mass contained in the simulation domain. The mass is locally preserved during collisions, because the zero order momentum of the collision operator is null. During the streaming process, the quantity $\rho r$ is propagated: the total mass being proportional to $\sum_{i, j}(\rho r)_{i j}$, it is exactly preserved by the streaming operator. Thus, despite the presence of truncation error terms in the continuity equation, the total mass of the system is exactly preserved by the model, provided that the boundary conditions are mass conservative.

\section{Axis boundary condition}

The axis boundary condition is performed on the axis boundary nodes. Those boundary nodes are located a half step ahead from the symmetry axis. To analyse the dynamic that is modelled by the axis boundary condition, we define the ghost nodes as the nodes located symmetrically to the boundary nodes with respect to the axis (Figure 1). Thus, the radius is $r=\Delta X / 2$ on the boundary nodes, it is $r=-\Delta X / 2$ on the ghost nodes.

The boundary condition should act as if the macroscopic flow was exactly symmetric on both sides of the axis. Let $\rho,\left(u_{r}, u_{z}\right)$, and $\left(a_{r}, a_{z}\right)$ be the density, velocity, and acceleration on a boundary node. Let $\tilde{\rho},\left(\tilde{u}_{r}, \tilde{u}_{z}\right)$, and $\left(\tilde{a}_{r}, \tilde{a}_{z}\right)$ be the same quantities on the corresponding ghost node. The boundary condition should act as if those quantities were equal to $\rho,\left(-u_{r}, u_{z}\right)$, and $\left(-a_{r}, a_{z}\right)$ (Figure 2).

The symmetry of the acceleration is already ensured by the definition of the axis force in Equation 10. Indeed the real force $F_{0 \alpha}$ is symmetric by definition, and the virtual force $F_{r}^{\prime}$ is an odd function of the radius $r$ and radial velocity $u_{r}$. If the symmetry is ensured for the density and the velocity, we get $\tilde{a}_{r}=-a_{r}$, and $\tilde{a}_{z}=a_{z}$.

The boundary condition is applied during the streaming process. Before the streaming process, distribution functions on the boundary node are post- 


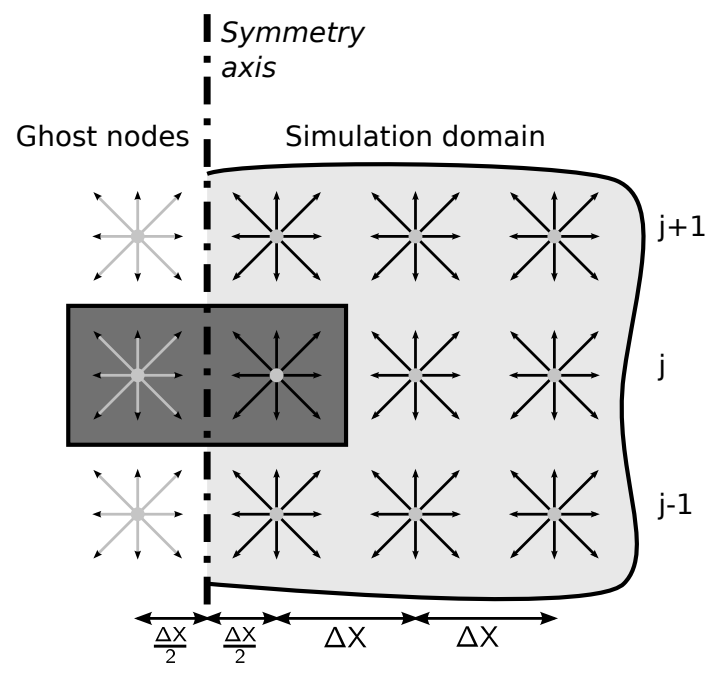

Figure 1: Boundary node and ghost node near the symmetry axis

collision distribution functions $f_{k}^{*}$. They are related to the node density $\rho$, velocities $\left(u_{r}, u_{z}\right)$ and accelerations $\vec{a}=\vec{F} / \rho$ through:

$$
\left\{\begin{array}{l}
\sum f_{k}^{*}=\frac{\Delta X}{2} \rho \\
\sum c_{k r} f_{k}^{*}=\frac{\Delta X}{2} \rho\left(u_{r}+\frac{\Delta t}{2} a_{r}\right) \\
\sum c_{k z} f_{k}^{*}=\frac{\Delta X}{2} \rho\left(u_{z}+\frac{\Delta t}{2} a_{z}\right)
\end{array}\right.
$$

\subsection{Mirror boundary conditions}

Guo [11] proposes a "mirror" type boundary condition (mirror BC) at the axis location. The mirror boundary condition allocates the outgoing distribution functions $f_{3}^{*}, f_{6}^{*}$ and $f_{7}^{*}$ to the surrounding unknown distribution functions (in the nodes $j-1, j$ and $j+1$ ) as follow:

$$
\left\{\begin{array}{l}
\left(f_{5}^{+}\right)_{j+1}=\left(f_{6}^{*}\right)_{j} \\
\left(f_{1}^{+}\right)_{j}=\left(f_{3}^{*}\right)_{j} \\
\left(f_{8}^{+}\right)_{j-1}=\left(f_{7}^{*}\right)_{j}
\end{array}\right.
$$




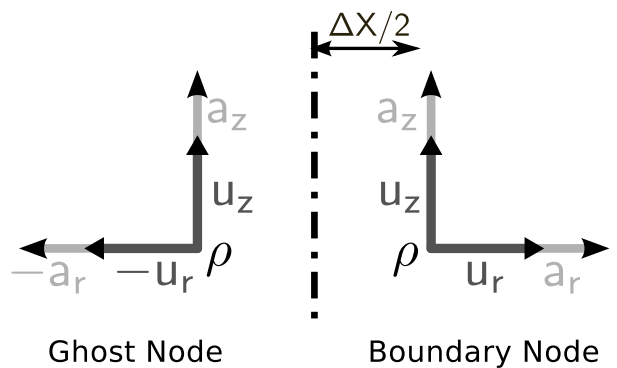

Figure 2: Target behaviour for the axis boundary condition

The mirror boundary condition acts as if, before the streaming, the ghost node distribution functions were a rotation around the vertical axis of tbhe boundary node distribution functions. This behaviour is represented in Figure 3.

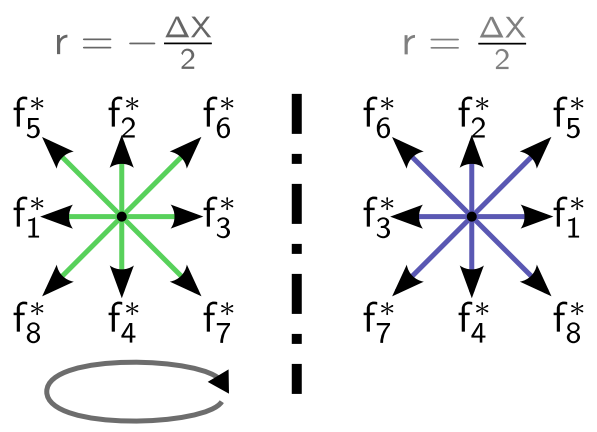

Figure 3: Ghost node distribution function for the mirror boundary condition

Now we can compute the macroscopic quantities $\tilde{\rho}$ and $\left(\tilde{u}_{r}, \tilde{u}_{z}\right)$ at the ghost node location before the streaming process. Recalling that the radius is $r=$ $-\Delta X / 2$, we get: 


$$
\left\{\begin{array}{l}
\tilde{\rho}=\frac{\sum f_{k}^{*}}{-\Delta X / 2}=-\rho \\
\left(\tilde{u}_{r}+\frac{\Delta t}{2} \tilde{a}_{r}\right)=\frac{-\sum c_{k r} f_{k}^{*}}{(-\Delta X / 2) \tilde{\rho}}=-\left(u_{r}+\frac{\Delta t}{2} a_{r}\right) \\
\left(\tilde{u}_{z}+\frac{\Delta t}{2} \tilde{a}_{z}\right)=\frac{\sum c_{k z} f_{k}^{*}}{(-\Delta X / 2) \tilde{\rho}}=\left(u_{z}+\frac{\Delta t}{2} a_{z}\right)
\end{array}\right.
$$

The symmetry is broken for the density. Although the ghost node velocities are correct, the mirror BC simulates opposite densities on both sides of the symmetry axis. It is not suited for the RW-LB model

\subsection{The radius weighted mirror boundary condition}

The radius weighted mirror boundary condition (RW Mirror BC) is a modified version of the mirror boundary condition which ensures the symmetry of the whole set of macroscopic quantities. To do so, we multiply by -1 the value of the distribution functions that cross the boundary;

$$
\left\{\begin{array}{l}
\left(\tilde{f}_{5}^{+}\right)_{j+1}=\left(-f_{6}^{*}\right)_{j} \\
\left(\tilde{f}_{1}^{+}\right)_{j}=\left(-f_{3}^{*}\right)_{j} \\
\left(\tilde{f}_{8}^{+}\right)_{j-1}=\left(-f_{7}^{*}\right)_{j}
\end{array}\right.
$$

The RW-mirror BC acts as if, before the streaming, the ghost node distribution functions were a rotation around the vertical axis of the boundary node distribution functions, and if all the values were multiplied by -1 . This behaviour is represented in Figure 3.

The macroscopic density and velocities now correspond to the targeted values:

$$
\left\{\begin{array}{l}
\tilde{\rho}=\frac{\sum\left(-f_{k}^{*}\right)}{-\Delta X / 2}=\rho \\
\left(\tilde{u}_{r}+\frac{\Delta t}{2} \tilde{a}_{r}\right)=\frac{-\sum c_{k r}\left(-f_{k}^{*}\right)}{(-\Delta X / 2) \tilde{\rho}}=-\left(u_{r}+\frac{\Delta t}{2} a_{r}\right) \\
\left(\tilde{u}_{z}+\frac{\Delta t}{2} \tilde{a}_{z}\right)=\frac{\sum c_{k z}\left(-f_{k}^{*}\right)}{(-\Delta X / 2) \tilde{\rho}}=\left(u_{z}+\frac{\Delta t}{2} a_{z}\right)
\end{array}\right.
$$




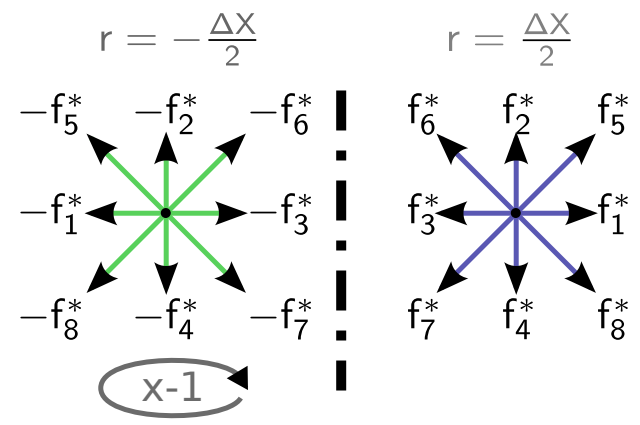

Figure 4: Ghost node distribution function for the RW-mirror boundary condition

However, the formulas from Equation 19 are not sufficient to get a good quality boundary conditions. Indeed, during the streaming process the domain looses $f_{3}^{*}+f_{6}^{*}+f_{7}^{*}$ from each boundary node and gets in return $-f_{3}^{*}-f_{6}^{*}-f_{7}^{*}$. There is a net mass loss $\rho^{\prime} r=2\left(f_{3}^{*}+f_{6}^{*}+f_{7}^{*}\right)$. This quantity must be re-injected into the simulation domain to ensure the mass conservation.

The mass correction is performed in such a way that the post-streaming velocities and accelerations are not affected. Just after the streaming, the mass at the boundary node is $\tilde{\rho} r=\sum \tilde{f}_{k}^{+}$. This mass must be modified to $\left(\tilde{\rho}+\rho^{\prime}\right) r$. We use:

$$
f_{k}^{+}=\tilde{f}_{k}^{+} \times\left(1+\frac{\rho^{\prime} r}{\tilde{\rho} r}\right)
$$

The RW-Mirror boundary condition involves two steps: a streaming process (Equation 19) and a mass correction (Equation 21). It ensures the symmetry of the macroscopic quantities, and the mass conservation in the simulation domain.

\subsection{Additional observations}

Axis symmetric simulations are normally performed on a simulation domain that covers a half section $[0, R]$ of the cylindrical domain, with proper boundary conditions near the symmetry axis. To observe the way the distribution 
functions are naturally arranged by the RW model around the axis location, we performed simulations on a whole section $[-R, R]$ of the cylindrical domain.

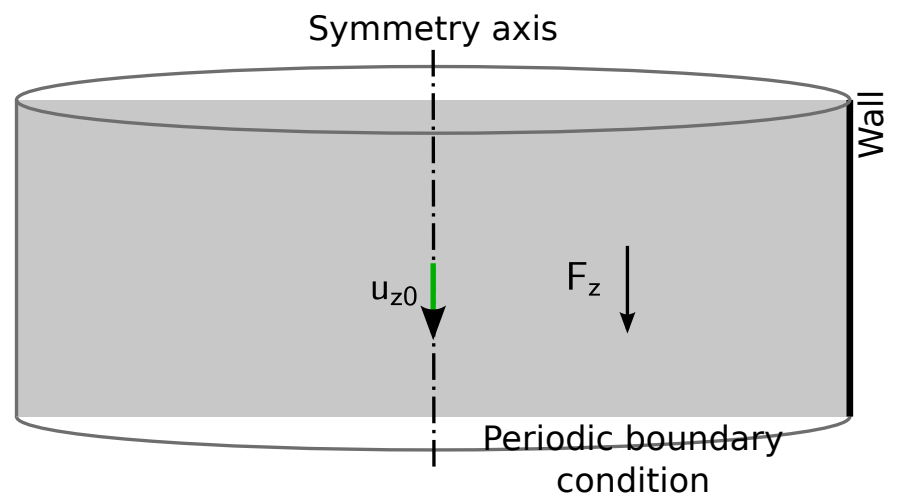

Figure 5: Simulation domain for the preliminary simulations of the Poiseuille flow

Two test cases were simulated: the Poiseuille Hagen flow (Figure 5), and the side driven cylinder. Those test cases are described in detail in sections 7.2 and 7.5. We compared the distribution functions on the nodes located near the axis: on the right side $(r=\Delta X / 2)$, and on the left side $(r=-\Delta X / 2)$. For both test cases, the relative arrangement between the left and right nodes is identical to the relative arrangement proposed by the RW-mirror boundary condition. The left node distribution functions were a rotation around the vertical axis of the right node distribution functions, with all the values multiplied by -1 (figure 4). The RW-mirror BC mimics the natural behaviour of the model near the axis.

Moreover, from the full section simulations, we could observe the effect of the error singularity as we approach the axis at $r=0$. The solution of the Poiseuille Hagen flow has a zero radial velocity: the singular term $\partial_{r}\left(u_{r} / r\right)$ in equation 14 is null. The simulation results were in good agreement with the analytical results, and no mass deviation was observed. However, the Side driven cavity flow implies radial velocity components: the singular term $\partial_{r}\left(u_{r} / r\right)$ in equation 14 is non null. We observed from the simulations a strong deviation of the total mass. 
Thus, the mass correction of the RW-mirror BC compensates the natural mass deviation caused by the singular error term in the continuity equation.

\section{Model implementation}

Dellar [27] interprets the $2^{\text {nd }}$ order LB scheme as a Strang Splitting procedure. Let $S$ be the streaming operator, and $C$ the collision operator. Dellar recommends the following sequence of operations for the computation of $N_{t}$ time-steps:

$$
f_{k}(n . \Delta t)=C^{1 / 2} S \overbrace{C S \ldots C S}^{\left(N_{t}-1\right) \times C . S .} C^{1 / 2}\left(f_{k}(0)\right)
$$

The initial density, velocity, and force are respectively $\rho_{\text {ini }}, \vec{u}_{\text {ini }}$ and $\vec{F}_{\text {ini }}$. At $t=0$, the distribution functions are at the equilibrium state: $f_{k}(0)=$ $f_{k}^{e q}\left(\rho_{\text {ini }}, \vec{u}_{\text {ini }}\right)$. After the first half-collision, we get:

$$
C^{1 / 2}\left(f_{k}(0)\right)=f_{k}^{e q}\left(\rho_{\mathrm{ini}}, \vec{u}_{\mathrm{ini}}\right)+\frac{\Delta t}{2} R_{k}\left(\vec{u}_{\mathrm{ini}}, \vec{F}_{0 \mathrm{ini}}+\vec{F}_{\mathrm{ini}}\right)
$$

where $\vec{F}^{\prime}$ ini is calculated, using Equation 10, with the initial density and radial velocity.

Then, the streaming and collision operators are applied successively $N_{t}-1$ times. The streaming operator $S$ splits-up into two steps:

- Normal streaming inside the computational domain

- Correction at the boundary nodes

We used half-way bounce-back boundary conditions along the solid walls. The collision operator $C$ splits-up into three steps:

- Calculation of the macroscopic quantities from pre-collision distribution functions. 
- Calculation of the equilibrium distribution functions (Equation 5), the virtual correcting force $\overrightarrow{F^{\prime}}$ (Equation 10), and the forcing terms $R_{k}$ (Equation 9)

- Collision (RHS of of Equation 4).

The determination of the macroscopic quantities $\rho$ and $\vec{u}$ is performed from pre-collision distribution functions $f_{k}$. The density is directly deduced from the zero-th order moment:

$$
\rho=\frac{\sum_{k} f_{k}}{r}
$$

For the velocity, it is slightly more complicated because the force term $\vec{F}=$ $\overrightarrow{F_{0}}+\overrightarrow{F^{\prime}}$ depends on the radial velocity $u_{r}$. The first order moment is inverted to get the velocities in the $r$ and $z$ directions:

$$
\begin{aligned}
& u_{r}=\frac{1}{r \rho+\Delta t \frac{\rho \nu}{r}}\left(\sum_{k} c_{k r} f_{k}+\frac{\Delta t}{2}\left(r F_{0 r}+\rho c_{s}^{2}\right)\right) \\
& u_{z}=\frac{1}{r \rho}\left(\sum_{k} c_{k z} f_{k}+\frac{\Delta t}{2} r F_{0 z}\right)
\end{aligned}
$$

At the end of the simulation, the available distribution functions are post collision distribution functions $f_{k}^{*}$. Their first order moment is different from Equation 7:

$$
\sum_{k} c_{k \alpha} f_{k}^{*}=r\left(\rho u_{\alpha}+\Delta t \frac{F_{\alpha}}{2}\right)
$$

Thus, the macroscopic velocities are obtained through:

$$
\begin{aligned}
& u_{r}=\frac{1}{r \rho-\Delta t \frac{\rho \nu}{r}}\left(\sum_{k} c_{k r} f_{k}^{*}-\frac{\Delta t}{2}\left(r F_{0 r}+\rho c_{s}^{2}\right)\right) \\
& u_{z}=\frac{1}{r \rho}\left(\sum_{k} c_{k z} f_{k}^{*}-\frac{\Delta t}{2} r F_{0 z}\right)
\end{aligned}
$$




\section{Test Cases}

The RW-LBM model is confronted to four test cases. The primary goal of those simulations is to compare the performances of the mirror boundary condition and the RW-mirror boundary conditions. The influence of the parameter $\tau$ on the model accuracy is investigated with the fourth test case.

The first test case is the Poiseuille Hagen flow. Then, we test the Wormersley flow because of its transitional behaviour. Those two test cases have analytical solutions with zero radial velocities. However, we emphasized the importance of the radial velocity on the error near the symmetry axis. So we investigate two more test cases: the Rayleigh Benard flow, and a made-up test case: the side driven cylinder.

\subsection{Stopping criterion}

The convergence error $\varepsilon_{c}$ is the error between two axial velocity fields separated by 500 time steps:

$$
\varepsilon_{c}=\sqrt{\frac{1}{N_{Z} \cdot N_{R}} \sum_{i} \sum_{j} \frac{\left|u_{z, i j}(t)-u_{z, i j}(t-500 \Delta t)\right|^{2}}{\left|u_{z, i j}(t)\right|^{2}}}
$$

Each simulation of a steady state system ended up with a convergence error below $10^{-10}$. For each of those simulations, the number of time steps we used is indicated through a non-dimension simulation time value $\kappa$. The definition of $\kappa$ depends on the simulated system.

The initial density is: $\rho_{0}=1$. The total mass deviation $\Delta M$ is expressed in 29. It did not exceed $10^{-15}$ during all the simulations. The mass conservation is ensured by the model.

$$
\Delta M=\frac{\sum_{i} \sum_{j} \rho_{i j} r_{i} \Delta X^{2}}{R^{2} H}-1
$$




\subsection{Poiseuille Hagen Flow}

The Poiseuille Hagen flow is a flow inside a vertical cylinder, driven by a homogeneous vertical force $\overrightarrow{F_{0}}=-F_{0 z} \cdot \overrightarrow{e_{z}}$. The simulated system is drawn in Figure 6. The symmetry axis is on the left. Half-way bounce-back boundary condition is applied on the right. Periodic boundary conditions are applied between the top and bottom limits of the domain.

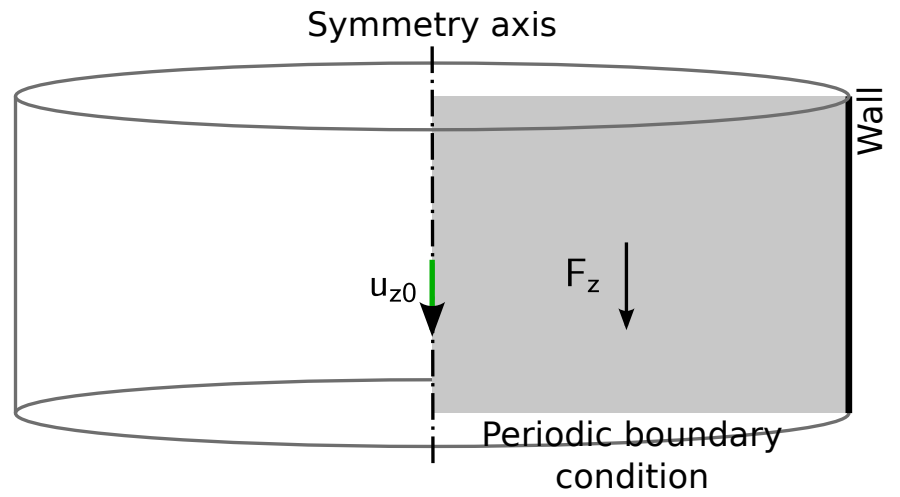

Figure 6: Simulation domain of the Poiseuille Hagen flow

The analytical solution is $\tilde{u}_{r}(r)=0$, and $\tilde{u}_{z}(r)$ given by Equation 30, where $U_{0}$ is the velocity at the axis location (Figure 7 ).

$$
\tilde{u}_{z}(r)=-U_{0}\left(1-\frac{r^{2}}{R^{2}}\right)=-\frac{F_{0 z} R^{2}}{4 \rho \nu}\left(1-\frac{r^{2}}{R^{2}}\right)
$$

The dynamics of a Poiseuille Hagen flow is fully characterized by the Reynolds number $R e=2 R U_{0} / \nu$. We used the following settings: $R e=10, \tau=0.75$, $\kappa=\left(N_{t} \Delta t\right) U_{0} / R=100, R=1$ and $\Delta t / \Delta X^{2}=1$. Density variations and horizontal velocities were close to the machine precision for all simulations.

The bottom axis of Figure 7 show the repartition of the axial velocity error along the radial coordinate, for the Mirror and the RW-Mirror boundary conditions. The error values are identical near the solid wall. They are due to the half way bounce-back scheme which, when used with a BGK model, causes a slight deviation of the wall location. This phenomenon is explained by Noble et al. [28] and Hecht and Harting [29]. 

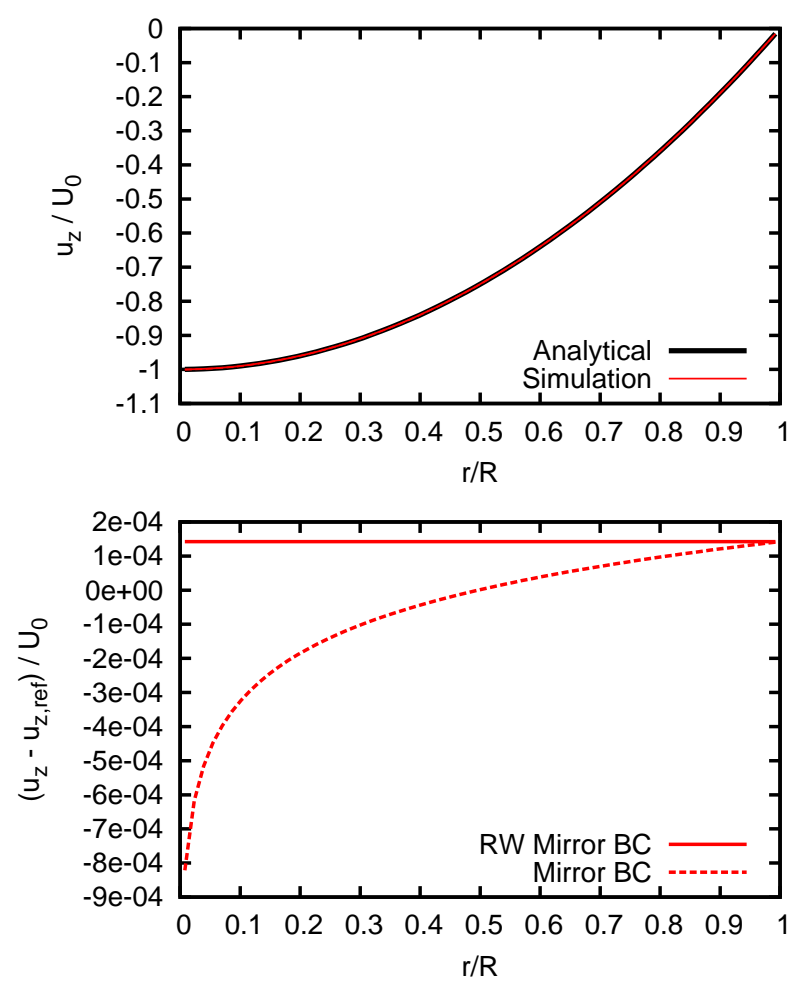

Figure 7: Poiseuille Hagen flow velocity profile for $N_{R}=64$ and $R e=10$. Bottom axis: distribution of the axial velocity error along the radial coordinate

With the RW-Mirror BC, the error keeps at the same level all across the flow stream. The errors due to the flow model and the axis $\mathrm{BC}$ are negligible compared to the initial error near the wall. The present result is very encouraging, because is assesses the consistency between the RW-LB model and the RW-Mirror boundary condition. Here, since there is no radial velocity, the mass correction in Equation 21 is null. The RW-Mirror BC reflects exactly the behaviour of the RW-LB model on the other side of the axis.

The error repartition which is obtained using the Mirror-BC is much worse. The error deviates strongly when approaching the axis.

To determine the degree of accuracy of the models, we compute the error $\varepsilon$ on the axis axial velocity: 


$$
\varepsilon=\frac{\left|U_{0}-u_{z 0}^{k}\right|}{U_{0}}
$$

The axial velocity $u_{z 0}=u_{z}(r=0)$ is extrapolated from the two neighbouring velocity values $u_{z, 0}$ and $u_{z, 1}$, with a second order extrapolation scheme assuming $\partial u_{z} / \partial r(0)=0$ :

$$
u_{z 0}=\frac{9}{8} u_{z, 0}-\frac{1}{8} u_{z, 1}
$$

The error $\varepsilon$ is represented as a function of the radial resolution $N_{R}$ in Figure 8. For both boundary conditions, we get $\varepsilon \propto \Delta X^{2}$, the model is second order accurate. The axis velocity predictions are more precise with the RW-Mirror $\mathrm{BC}$ for all mesh sizes.

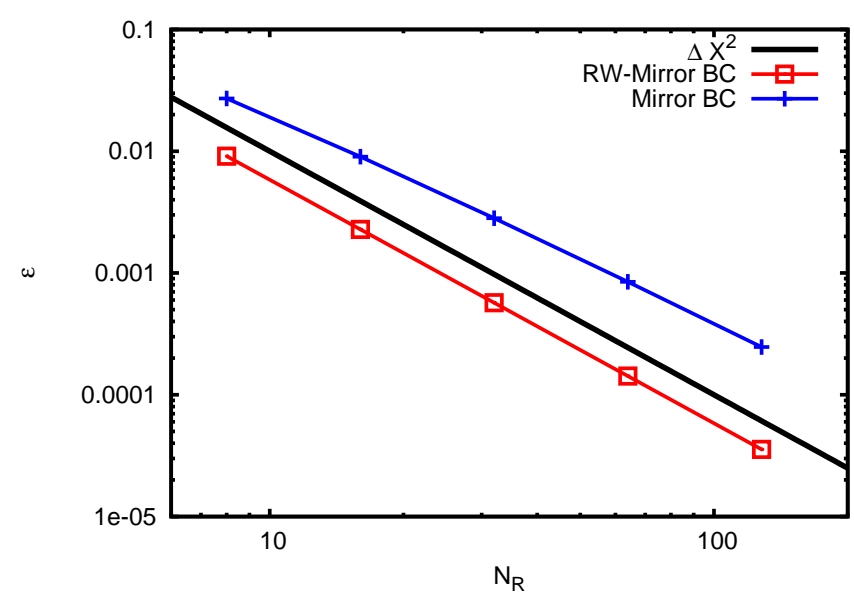

Figure 8: Grid dependency on the axis velocity for the Poiseuille flow

\subsection{Womersley Flow}

The Womersley flow is the flow inside a tube, driven by an oscillatory axial force:

$$
\overrightarrow{F_{0}}=-F_{0 z} \cos (\omega \cdot t) \overrightarrow{e_{z}}
$$


The reference velocity is $U_{0}=F_{0 z} R^{2} /(4 \rho \nu)$. The dynamic of the systems depends on the Reynolds number $R e=2 R U_{0} / \nu$ and the Womersley number $\alpha=$ $R \sqrt{\omega / \nu}$. The analytical solution has a zero radial velocity, and an axial velocity given by Equation 34, where $J_{0}$ is the zero order complex Bessel function.

$$
\tilde{u}_{z}(r, t)=\operatorname{abs}\left[\frac{4 U_{0}}{\alpha^{2} i}\left(1-\frac{J_{0}(\sqrt{i \omega / \nu} \cdot r)}{J_{0}(\sqrt{i \omega / \nu} \cdot R)}\right) \exp (i \omega t)\right]
$$

The parameters we used for the simulations are: $R e=1200, \alpha=20, \tau=0.6$, $R=1$ and $\Delta t / \Delta X^{2}=1$. The simulations were run over 400 periods to reach the periodic regime.

Simulated axial velocity profiles are drawn for four moments of the oscillatory process in Figures 9 (mirror boundary conditions) and 10 (radius weighted mirror boundary conditions). In each figure, the simulation results are confronted to the theoretical profiles. In Figure 9, the mirror boundary causes a deviation of the velocity profile near $r=0$ (circled area). This deviation is null with the Radius weighted boundary condition.

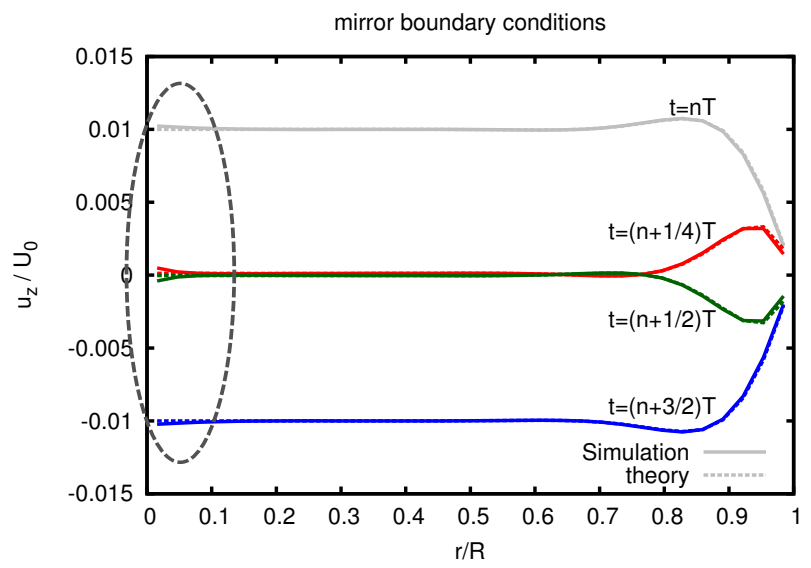

Figure 9: Typical solution for the wormersley flow with $\alpha=20$. Mirror boundary conditions $\left(N_{R}=32\right)$

The error $\varepsilon$ was computed from the axial velocity during the last simulated period: 


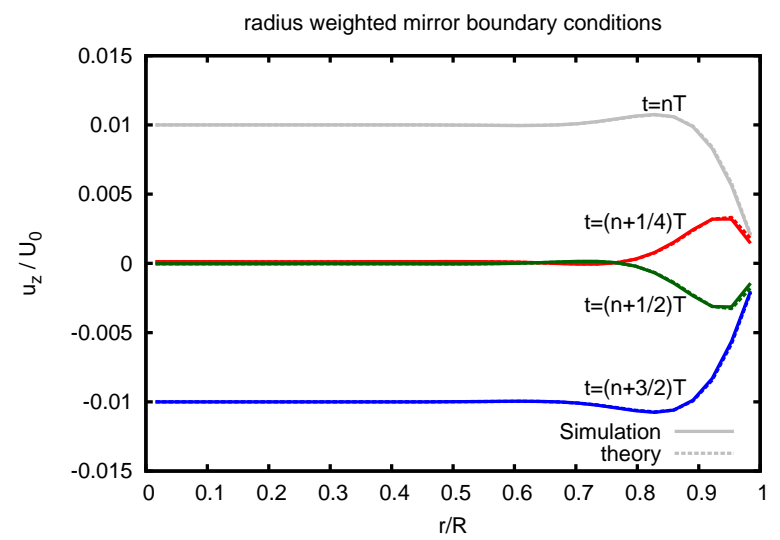

Figure 10: Typical solution for the wormersley flow with $\alpha=20$. RW Mirror boundary conditions $\left(N_{R}=32\right)$

$$
\varepsilon=\frac{1}{N t_{T}} \sum_{k}^{N t_{T}} \frac{\left|\tilde{u}_{z}\left(0, t_{k}\right)-u_{z 0}^{k}\right|}{\left|\tilde{u}_{z}\left(0, t_{k}\right)\right|}
$$

It is represented as a function of the radial resolution $N_{R}$ in Figure 11. It is difficult to extract any order accuracy from those curves. The precision is generally improved during the mesh refinement. Axis velocity predictions are on average 500 times more precise with the RW-mirror BC.

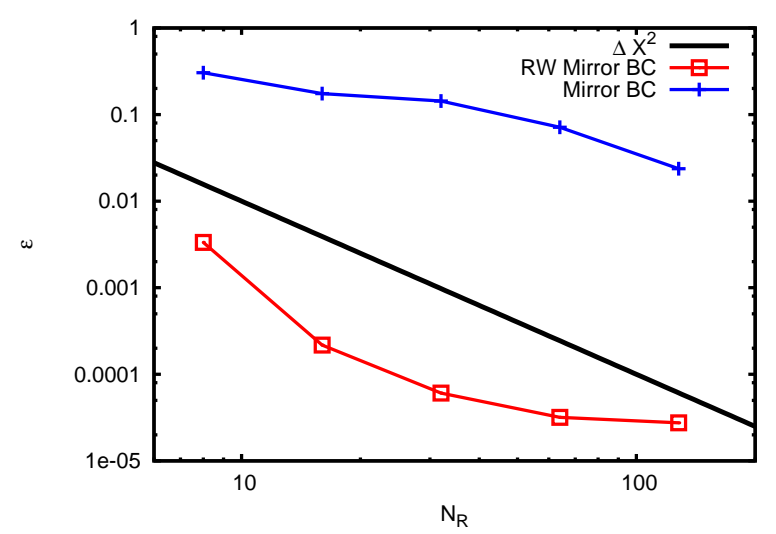

Figure 11: Error on the axis velocity for the Wormersley flow 


\subsection{Rayleigh Benard convection}

The choice of the Rayleigh Benard convection flow is motivated by the presence of non-zero radial velocity, and the existence of reference results in the literature.

The fluid, initially at rest, is enclosed inside a vertical cylinder, with a hot temperature $T_{h}$ at the bottom, a cold temperature $T_{c}$ at the top, and adiabatic conditions on the surrounding wall (Figure 12). The Boussinesq force $\vec{F}_{0}=$ $\rho g \beta\left(T-T_{m}\right) \overrightarrow{e_{z}}$ drags a toroidal flow inside the cylinder.

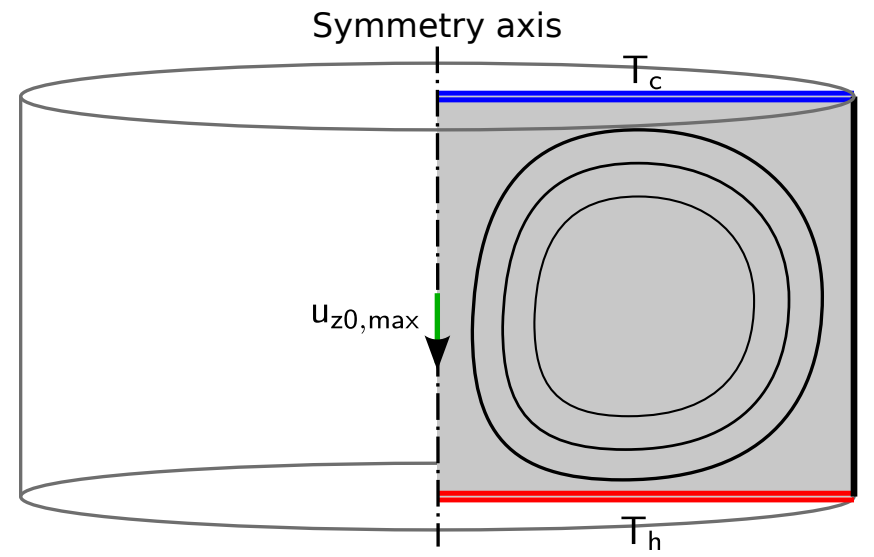

Figure 12: Simulated system for the Rayleigh Benard convection

The flow model is coupled with an energy conservation model. The energy conservation model was built on the same grid than the flow model. At each time step, the temperature values on the lattice sites are updated using Equation 36. The spatial derivatives $\tilde{\partial}_{r}, \tilde{\partial}_{z}, \tilde{\partial}_{r}^{2}$ and $\tilde{\partial}_{z}^{2}$ are centred discrete derivatives. They are second order accurate in $\Delta X$.

$$
T^{k+1}=T^{k}+\Delta t\left[-u_{r} \tilde{\partial}_{r} T^{k}-u_{z} \tilde{\partial}_{z} T^{k}+\alpha\left(\tilde{\partial}_{r}^{2} T^{k}+\tilde{\partial}_{z}^{2} T^{k}+\frac{\tilde{\partial}_{r} T^{k}}{r}\right)\right]
$$

The reference velocity is $U_{0}=\sqrt{g \beta\left(T_{h}-T_{c}\right) H}$. The following criteria were fulfilled in order to ensure the stability of the energy conservation model (see Tannehill et al. [30]): 
- Diffusive criterion: $\frac{\alpha \Delta t}{\Delta X^{2}} \leq \frac{1}{4}$

- Two dimensions Courant Friedrichs Lewi criterion: $\frac{U_{0} \Delta t}{\Delta X} \leq \frac{1}{2}$

- Mixed criterion: $\frac{U_{0}^{2} \Delta t}{\alpha} \ll 1$

The dynamics of the system is fully characterized by three dimensionless numbers: Rayleigh $R a=\frac{g \beta\left(T_{h}-T_{c}\right) H^{3}}{\alpha \nu}$, Prandtl $\operatorname{Pr}=\frac{\nu}{\alpha}$, and shape factor $\Gamma=R / H$. The parameters we used were: $R a=5000, \operatorname{Pr}=0.7, \Gamma=1$, $\tau=0.55, R=1, \Delta t / \Delta X^{2}=1$ and $\kappa=\alpha N_{t} \Delta t / R^{2}=14$.

The reference result given by Lemembre and Petit [31] is the maximum dimensionless axis velocity: $u_{z 0, \max } / U_{0}=0.351$. The small number of digits of this reference result is due to the weakly oscillatory nature of the toroidal flow. The simulation results are compared to the reference value in Figure 13. The models with both boundary conditions converge toward the right velocity value. However, the precision is far better with the RW-mirror BC than with the mirror BC.

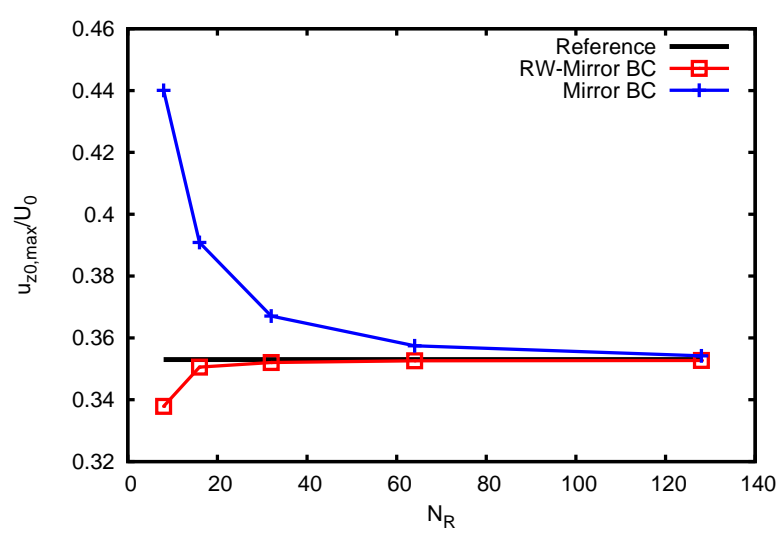

Figure 13: Maximum axis velocity for Rayleigh Benard convection

The direction of the fluid rotation depends on the initial temperature $T^{\text {ini }}$.

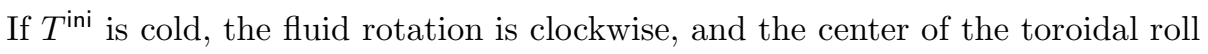
is slightly shifted upward. If $T^{\text {ini }}$ is hot, the cold top temperature implies a decrease of the buoyancy: the rotation is anti-clockwise, and the center of the toroidal roll is shifted downward. This phenomenon was correctly reproduced 
by the RW LMB model. Velocity streamlines are drawn on Figure 14(a) for an initial cold temperature, and on Figure 14(b) for an initial hot temperature.

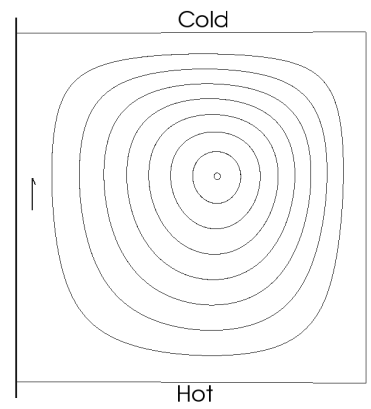

(a)

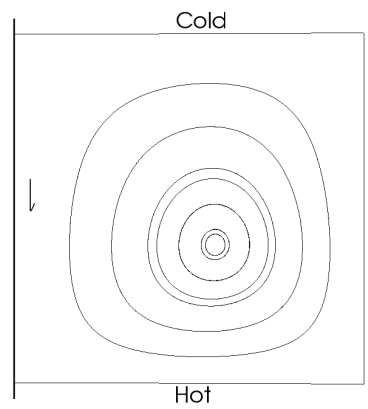

(b)

Figure 14: Velocity streamlines for Rayleigh-Benard convection:14(a) initial cold temperature, 14(b) initial hot temperature

\subsection{Side driven cylinder}

To fully characterize the RW LBM model, we need a test case with a non zero radial velocity, which does not require any coupling with other conservation models. That's why we investigate the side driven cylinder flow. The side driven cylinder is a vertical cylinder with solid walls on the top and bottom surfaces. The fluid is driven by an axial velocity $U_{0}$ which is imposed on the side of the cylinder (see fig. 15) 


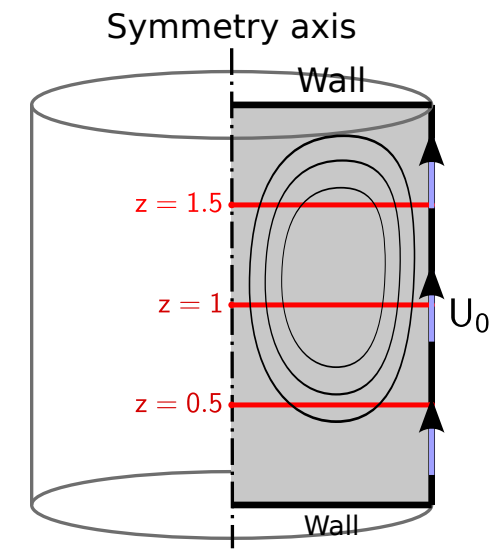

Figure 15: Simulated system for the side driven cavity

We fix $z=0$ at the bottom of the system. A weighting is applied on the side wall velocity to avoid discontinuity issues near the corners.

$$
U_{0}^{\prime}(z)=U_{0} \times\left[1-\left(\frac{2 z}{H}-1\right)^{18}\right]^{2}
$$

The dynamic of the system is entirely defined by two dimensionless numbers: Reynolds number $R e=U_{0} H / \nu$, and a shape factor: $\Gamma=H / R$. We performed the simulations for $R e=50$ and $\Gamma=2$, with the following settings: $\tau=0.6$, $R=1, \Delta t / \Delta X^{2}=1$, and $\kappa=N_{t} \Delta t U_{0} / H=30$.

We got the reference velocity values from much finer simulations performed on a finite volume model based on the axis-symmetric Navier-Stokes equations. For those simulations, we used a steady state model, with a pressure-velocity coupling scheme which offers a second order accuracy for the pressure, and a third order accuracy for the momentum, through the MUSCLE scheme. The reference results $\left(\tilde{u}_{r}, \tilde{u}_{z}\right)$ were obtained with a regular square mesh of $1024 \times 2048$ cells. A fourth order spline interpolation technique was used to move reference and LBM velocity values to the same locations for comparisons.

The lines $z=0.5, z=1$ and $z=1.5$ are identified in Figure 15. The radial and axial velocity profiles along those lines are represented in Figure 16, where 
the reference results are drawn in black. Absolute error repartitions are drawn in the bottom axis.

The error values are much greater for the side driven cavity flow (around 10-15\% with $N_{R}=64$ ) than for the Poiseuille Hagen flow (less than $0.1 \%$, see fig. 7). The present test case is stiffer, due to non-null radial velocity, and velocity gradients in both radial and axial directions.

The distinction between Mirror and RW-mirror BC is perceptible near the axis (circled areas). With the RW-Mirror BC, the radial velocity error tends toward zero and the axial velocity error stabilizes. With the Mirror BC, there is a distortion of the error profiles near the axis.
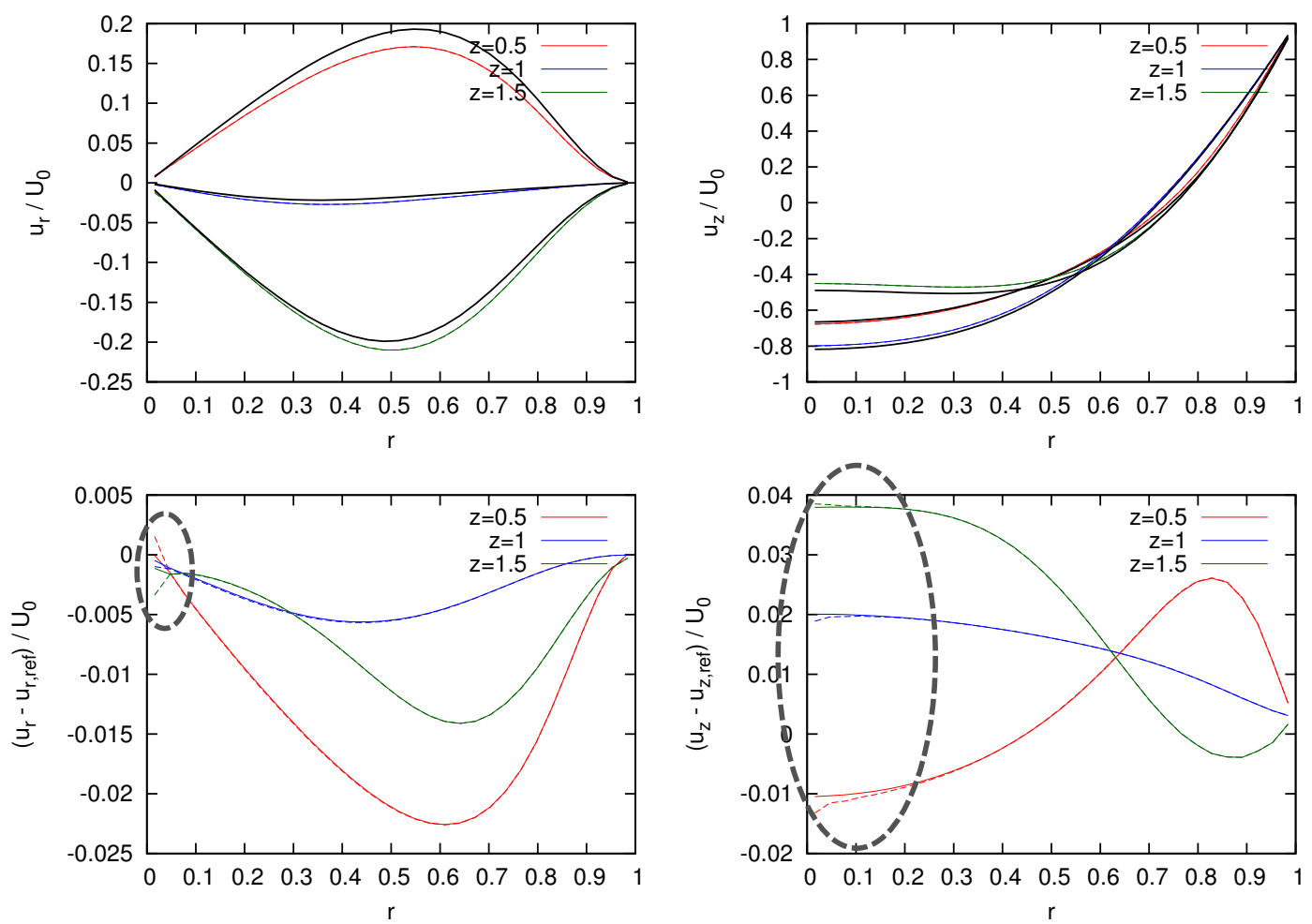

Figure 16: Axial and radial velocity profiles along the lines $y=0.5, y=1$ and $y=1.5$, for $N_{R}=64$ and $R e=50$. Reference profiles are drawn in black, simulated profiles with the RW-mirror and mirror boundary conditions are respectively in solid lines and dashed lines. The corresponding error are reported on the curves below. 
The global simulation error $\varepsilon$ is computed from the axial velocity profiles at the axis location $r=0$ (Equation 38), where $u_{z 0, j}$ are extrapolated values (Equation 32).

$$
\varepsilon=\frac{1}{N_{Z}} \sum_{j} \frac{\left|u_{z 0, j}-\tilde{u}_{z 0, j}\right|}{U_{0}}
$$

The error $\varepsilon$ is drawn in figure 17 for several mesh sizes. The convergence is now first order. This is an issue, because the truncation error analysis does not highlight any first order error term. It seems that the spurious terms $u_{r} / r$ become so great near the axis that they influence the convergence rate of the model.

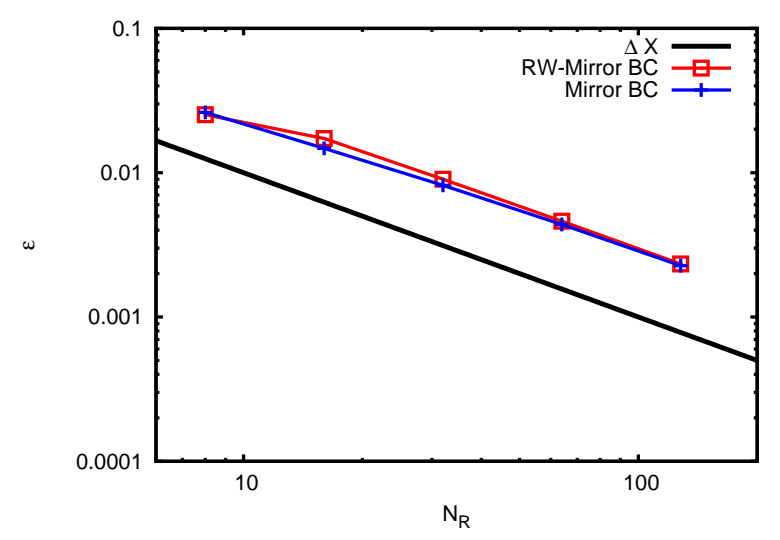

Figure 17: Axis axial velocity error for the side driven cylinder flow

The effect of the relaxation parameter $\tau$ is investigated in Figure 18. We set $R=1, U_{0}=1, N_{R}=32$ and $\nu=2 / R e$, with $R e=5, R e=10$ or $R e=100$. Since $\nu$ is fixed and $\tau$ is varying, the ratio $\Delta t / \Delta X^{2}$ is varying from one simulation to another.

For high Reynolds numbers ( $R e=50, R e=100)$, the trend is clear: the closer is the relaxation parameter to 0.5 , the more precise is the model. Here, the value $\nu=1 / R e$ being very small, the dominant error terms in Equations 14 and A.37 are multiples of $(2 \tau-1)$. The error is minimized when $\tau$ approaches 
0.5 .

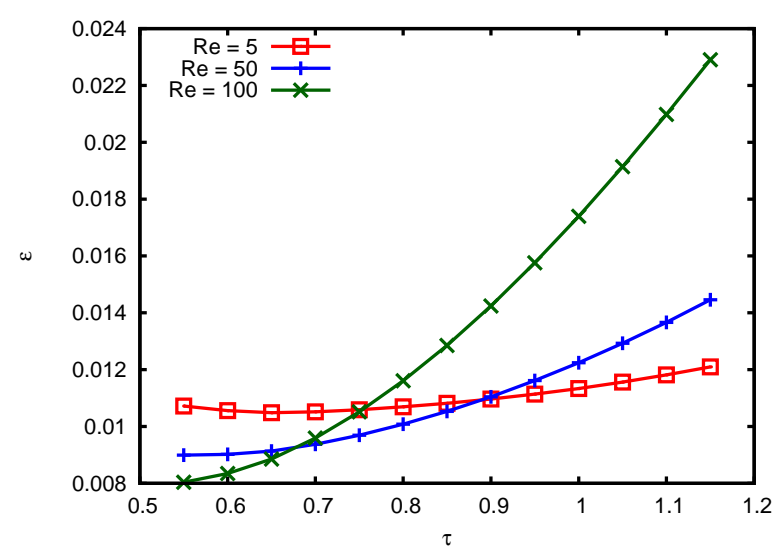

Figure 18: Effect of the relaxation parameter $\tau$ on the model precision.

For $R e=5$, the minimal error occurs near $\tau=6.5$. The dominant error terms in 14 and A.37 are no more the multiples of $(2 \tau-1)$, because the terms in $6 \tau^{2}-6 \tau+1($ root $\tau \approx 0.789)$ and $12 \tau^{2}-12 \tau+1$ (root $\tau \approx 0.908$ ) are getting bigger. The optimal value of $\tau$ is then shifted upward. This observation is in concordance with the analysis of the classical BGK model made by Holdych [8].

\section{Conclusions}

The present paper offers a deep and critical analysis of the RW-LB model, based the expression of its generalized equation. The generalized equation assesses the consistency of the model. All the error terms are multiples of $\Delta X^{2}$ or more: the model is second order accurate. However, we identified spurious error terms $u_{r} / r$ which may considerably reduce the model precision when $u_{r}$ is not null.

The fact that the spurious terms might reach extreme values near the axis motivated an investigation about the axis boundary condition. This investigation resulted in the definition of a new boundary condition which mimics the natural behaviour of the model when $u_{r}=0$, and compensates the mass loss 
when $u_{r} \neq 0$. The new boundary condition clearly provides more accurate simulation results.

The spurious error terms have no effect on the simulations results when $u_{r}=0$. We recovered the second order accuracy that we expected from the generalized equation. However, our last simulation results showed that strong values of $u_{r}$ reduced the order of accuracy of the model. We still don't know how to quantify this reduction, this is a work to be continued.

Anyway, despite those limitations, the RW-LB model still has the great advantage over any other model that the collision process is purely local. It remains an alternative to any Navier Stokes discretization when an exceptional precision is not necessarily needed.

\section{Acknowledgments}

We would like to thank the French agency ANR for its financial support through the project Stock-E MICMCP.

\section{Appendix A. Taylor series analysis}

\section{Appendix A.1. Native Expression}

The first step of the Taylor series analysis is to build the native expression of the model. The native expression is when the distribution functions $f_{k}(\vec{x}, t)$ are expressed as a function of macroscopic quantities $\rho, p, \vec{u}$, and $\vec{F}$ at the time $t$ and coordinate $\vec{x}$.

To distinguish the time step $\Delta t$ and space step $\Delta X$, the particle velocities $\overrightarrow{c_{k}}$ are formulated as the product of an amplitude $\Delta X / \Delta t$ and a direction vector $\overrightarrow{w_{k}}$. For the sake of clarity, we adopt the following formulation:

$$
f_{k}(n)=f_{k}\left(\vec{x}+n \overrightarrow{c_{k}} \Delta t, t+n \Delta t\right)=f_{k}\left(\vec{x}+n \Delta X \vec{w}_{k}, t+n \Delta t\right)
$$

We start with the LBM Equation 4: 


$$
f_{k}(1)-f_{k}=-\frac{1}{\tau}\left(f_{k}-f_{k}^{e q}\right)+\Delta t\left(1-\frac{1}{2 \tau}\right) R_{k}
$$

By simply shifting the time continuum by $-\Delta t$, we get:

$$
f_{k}=\left(1-\frac{1}{\tau}\right) f_{k}(-1)+\frac{1}{\tau} f_{k}^{e q}(-1)+\Delta t\left(1-\frac{1}{2 \tau}\right) R_{k}(-1)
$$

This expression is applied recursively over an infinite number of time steps. By this process, all the unknown distribution functions $f_{k}(-n)$ are replaced by equilibrium distribution functions $f_{k}^{e q}(-n)$ and source terms $R_{k}(-n)$ :

$$
\begin{aligned}
& f_{k}=\frac{1}{\tau} \sum_{n=1}^{\infty}\left(1-\frac{1}{\tau}\right)^{n-1} f_{k}^{e q}(-n)+ \\
& \Delta t\left(1-\frac{1}{2 \tau}\right) \sum_{n=1}^{\infty}\left(1-\frac{1}{\tau}\right)^{n-1} R_{k}(-n)
\end{aligned}
$$

$f_{k}^{e q}$ and $R_{k}$ are directly related to the macroscopic quantities $\rho, p, \vec{u}$, and $\vec{F}$. Equation A.4 expresses $f_{k}(\vec{x}, t)$ as a function of the macroscopic quantities at the previous time steps. We use Taylor series expansion to express them at the present time:

$$
\begin{aligned}
& f_{k}^{e q}(-n)=f_{k}^{e q}+\sum_{m=1}^{\infty} \frac{1}{m !}\left[-n \Delta t \partial_{t}-n \Delta X \overrightarrow{w_{k}} \cdot \vec{\nabla}\right]^{m} f_{k}^{e q} \\
& R_{k}(-n)=R_{k}+\sum_{m=1}^{\infty} \frac{1}{m !}\left[-n \Delta t \partial_{t}-n \Delta X \overrightarrow{w_{k}} \cdot \vec{\nabla}\right]^{m} R_{k}
\end{aligned}
$$

Those expressions are introduced in A.4:

$$
\begin{aligned}
f_{k}= & \frac{1}{\tau} \sum_{n=1}^{\infty}\left(1-\frac{1}{\tau}\right)^{n-1} f_{k}^{e q}+\Delta t\left(1-\frac{1}{2 \tau}\right) \sum_{n=1}^{\infty} \sum_{m=1}^{\infty}\left(1-\frac{1}{\tau}\right)^{n-1} R_{k} \\
& +\frac{1}{\tau} \sum_{n=1}^{\infty} \sum_{m=1}^{\infty}\left(1-\frac{1}{\tau}\right)^{n-1} \frac{(-n)^{m}}{m !}\left[\Delta t \partial_{t}+\Delta X \overrightarrow{w_{k}} \cdot \vec{\nabla}\right]^{m} f_{k}^{e q} \\
& +\Delta t\left(1-\frac{1}{2 \tau}\right) \sum_{n=1}^{\infty}\left(1-\frac{1}{\tau}\right)^{n-1} \frac{(-n)^{m}}{m !}\left[\Delta t \partial_{t}+\Delta X \overrightarrow{w_{k}} \cdot \vec{\nabla}\right]^{m} R_{k}
\end{aligned}
$$


We introduce the function $p[\tau, m]$ which is defined as:

$$
p[\tau, m]=\frac{1}{\tau^{2}} \sum_{n=1}^{\infty}\left(1-\frac{1}{\tau}\right)^{n-1}(-n)^{m}
$$

The function $p[\tau, m]$ has a formal expression for the first values of $m$ :

$$
\begin{aligned}
& p[\tau, 0]=1 / \tau \\
& p[\tau, 1]=-1 \quad p[\tau, 2]=2 \tau-1 \\
& p[\tau, 3]=-6 \tau^{2}+6 \tau-1 \quad p[\tau, 4]=24 \tau^{3}-36 \tau^{2}+14 \tau-1
\end{aligned}
$$

Equation A.6 is then reduced to the following expression:

$$
\begin{aligned}
f_{k}= & f_{k}^{e q}+\Delta t\left(\tau-\frac{1}{2}\right) R_{k} \\
& +\tau \sum_{m=1}^{\infty} \frac{p[\tau, m]}{m !}\left[\Delta t \partial_{t}+\Delta X \overrightarrow{w_{k}} \cdot \vec{\nabla}\right]^{m} f_{k}^{e q} \\
& +\Delta t \tau \frac{(2 \tau-1)}{2} \sum_{m=1}^{\infty} \frac{p[\tau, m]}{m !}\left[\Delta t \partial_{t}+\Delta X \overrightarrow{w_{k}} \cdot \vec{\nabla}\right]^{m} R_{k}
\end{aligned}
$$

Finally, we expand the global differential operators:

$$
\left[\Delta t \partial_{t}+\Delta X \overrightarrow{w_{k}} . \vec{\nabla}\right]^{m}=\sum_{q=0}^{m} \frac{m !}{q !(m-q) !} \Delta t^{m-q} \Delta X^{q}\left[\partial_{t}\right]^{m-q}\left(\overrightarrow{w_{k}} \cdot \vec{\nabla}\right)^{q}
$$

The previous equation becomes:

$$
\begin{aligned}
& f_{k}=f_{k}^{e q}+\Delta t \frac{(2 \tau-1)}{2} R_{k} \\
& +\Delta t \tau \sum_{m=1}^{\infty} \sum_{q=0}^{m} \frac{p[\tau, m]}{q !(m-q) !} \Delta t^{m-q-1} \Delta X^{q}\left[\partial_{t}\right]^{m-q}\left(\overrightarrow{w_{k}} \cdot \vec{\nabla}\right)^{q} f_{k}^{e q} \\
& \quad+\Delta t \tau \sum_{m=1}^{\infty} \sum_{q=0}^{m} \frac{p[\tau, m]}{q !(m-q) !}\left(\frac{2 \tau-1}{2}\right) \Delta t^{m-q} \Delta X^{q}\left[\partial_{t}\right]^{m-q}\left(\overrightarrow{w_{k}} \cdot \vec{\nabla}\right)^{q} R_{k}
\end{aligned}
$$


In Equation A.11, the distribution functions $f_{k}(\vec{x}, t)$ are entirely defined from the macroscopic quantities at the time $t$ and the coordinate $\vec{x}$. We kept one term $\tau \Delta t$ at the beginning of each sum to simplify subsequent calculations.

Appendix A.2. Explicit expressions of the equilibrium distribution functions and force terms

To select the dominant terms in Equation A.11, we must expand the expression of $f_{k}^{e q}$ and $R_{k}$ as functions of $\Delta t$ and $\Delta X$. For that purpose, we consider $c_{s}^{2}=\Delta X^{2} / 3 \Delta t^{2}$ and $\overrightarrow{c_{k}}=(\Delta X / \Delta t) \overrightarrow{w_{k}}$.

The first element of $f_{k}^{e q}$ is directly expressed as a function of the pressure $p$ through the density / pressure relationship for incompressible flows:

$$
\rho=\frac{p}{c_{s}^{2}}=\frac{3 \Delta t^{2}}{\Delta X^{2}} p
$$

With this artefact, the pressure $p$ appears naturally in the momentum equation. We expand $f_{k}^{e q}$ and $R_{k}$ :

$$
\begin{aligned}
f_{k}^{e q} & =\omega_{k} r\left[\frac{p}{c_{s}^{2}}+\rho \frac{\overrightarrow{c_{k}} \cdot \vec{u}}{c_{s}^{2}}+\rho \frac{\left(\overrightarrow{c_{k}} \cdot \vec{u}\right)^{2}}{2 c_{s}^{4}}-\rho \frac{\vec{u}^{2}}{2 c_{s}^{2}}\right] \\
& =\omega_{k} r\left[\frac{3 \Delta t^{2}}{\Delta X^{2}} p+\frac{3 \Delta t}{\Delta X} \rho \overrightarrow{w_{k}} \cdot \vec{u}+\frac{9 \Delta t^{2}}{2 \Delta X^{2}} \rho\left(\overrightarrow{w_{k}} \cdot \vec{u}\right)^{2}-\frac{3 \Delta t^{2}}{2 \Delta X^{2}} \rho \vec{u}^{2}\right] \\
& =\frac{\Delta t^{2}}{\Delta X^{2}}\left[3 \omega_{k} r p+\frac{9 \omega_{k}}{2} \rho r\left(\overrightarrow{w_{k}} \cdot \vec{u}\right)^{2}-\frac{3 \omega_{k}}{2} \rho r \vec{u}^{2}\right]+\frac{\Delta t}{\Delta X}\left[3 \omega_{k} \rho r \overrightarrow{w_{k}} \cdot \vec{u}\right] \\
& =\frac{\Delta t^{2}}{\Delta X^{2}}\left[A_{f k}\right]+\frac{\Delta t}{\Delta X}\left[B_{f k}\right]
\end{aligned}
$$




$$
\begin{aligned}
R_{k} & =\omega_{k} r\left[\frac{\overrightarrow{c_{k}} \cdot \vec{F}}{c_{s}^{2}}+\frac{\left(\overrightarrow{c_{k}} \cdot \vec{u}\right)\left(\overrightarrow{c_{k}} \cdot \vec{F}\right)}{c_{s}^{4}}-\frac{\vec{u} \cdot \vec{F}}{c_{s}^{2}}\right] \\
& =\omega_{k} r\left[\frac{3 \Delta t}{\Delta X} \overrightarrow{w_{k}} \cdot \vec{F}+\frac{9 \Delta t^{2}}{\Delta X^{2}}\left(\overrightarrow{w_{k}} \cdot \vec{u}\right)\left(\overrightarrow{w_{k}} \cdot \vec{F}\right)-\frac{3 \Delta t^{2}}{\Delta X^{2}} \vec{u} \cdot \vec{F}\right] \\
& =\frac{\Delta t^{2}}{\Delta X^{2}}\left[9 \omega_{k} r\left(\overrightarrow{w_{k}} \cdot \vec{u}\right)\left(\overrightarrow{w_{k}} \cdot \vec{F}\right)-3 \omega_{k} r \vec{u} \cdot \vec{F}\right]+\frac{\Delta t}{\Delta X}\left[3 \omega_{k} r \overrightarrow{w_{k}} \cdot \vec{F}\right] \\
& =\frac{\Delta t^{2}}{\Delta X^{2}}\left[A_{R k}\right]+\frac{\Delta t}{\Delta X}\left[B_{R k}\right]
\end{aligned}
$$

In both expanded expressions, we get some terms that are multiples of $\Delta t^{2} / \Delta X^{2}$, and on term which is a multiple of $\Delta t / \Delta X$. The $\Delta t^{2} / \Delta X^{2}$ elements contain an even number of $\overrightarrow{w_{k}}$. The $\Delta t / \Delta X$ element contain and odd number of $\overrightarrow{w_{k}}$. This distinction is important given that the odd-order momentums in $\overrightarrow{w_{k}}$ are null. For the even order moments:

$$
\begin{aligned}
& \sum_{k} \omega_{k}=1 \\
& \sum_{k} \omega_{k} w_{k \alpha} w_{k \beta}= \begin{cases}1 / 3 & \text { if } 2 r \text { or } 2 z \\
0 & \text { if } r z\end{cases} \\
& \sum_{k} \omega_{k} w_{k \alpha} w_{k \beta} w_{k \gamma} w_{k \tau}= \begin{cases}1 / 3 & \text { if } 4 r \text { or } 4 z \\
0 & \text { if } r 3 z \text { or } z 3 r \\
1 / 9 & \text { if } 2 z 2 r\end{cases} \\
& \sum_{k} \omega_{k} w_{k \alpha} w_{k \beta} w_{k \gamma} w_{k \tau} w_{k \xi} w_{k \kappa}= \begin{cases}1 / 3 & \text { if } 6 r \text { or } 6 z \\
0 & \text { if } r 5 z \text { or } z 5 r \text { or } 3 r 3 z \\
1 / 9 & \text { if } 4 r 2 z \text { or } 4 z 2 r\end{cases}
\end{aligned}
$$

We get from A.11: 


$$
\begin{aligned}
& f_{k}=f_{k}^{e q}+\Delta t \frac{(2 \tau-1)}{2} R_{k} \\
& +\Delta t \tau \sum_{m=1}^{\infty} \sum_{q=0}^{m} \frac{p[\tau, m]}{q !(m-q) !} \Delta t^{m-q+1} \Delta X^{q-2}\left[\partial_{t}\right]^{m-q}\left(\overrightarrow{w_{k}} \cdot \vec{\nabla}\right)^{q} A_{f k} \\
& +\Delta t \tau \sum_{m=1}^{\infty} \sum_{q=0}^{m} \frac{p[\tau, m]}{q !(m-q) !}\left(\frac{2 \tau-1}{2}\right) \Delta t^{m-q+2} \Delta X^{q-2}\left[\partial_{t}\right]^{m-q}\left(\overrightarrow{w_{k}} \cdot \vec{\nabla}\right)^{q} A_{R k} \\
& +\Delta t \tau \sum_{m=1}^{\infty} \sum_{q=0}^{m} \frac{p[\tau, m]}{q !(m-q) !} \Delta t^{m-q} \Delta X^{q-1}\left[\partial_{t}\right]^{m-q}\left(\overrightarrow{w_{k}} \cdot \vec{\nabla}\right)^{q} B_{f k} \\
& +\Delta t \tau \sum_{m=1}^{\infty} \sum_{q=0}^{m} \frac{p[\tau, m]}{q !(m-q) !}\left(\frac{2 \tau-1}{2}\right) \Delta t^{m-q+1} \Delta X^{q-1}\left[\partial_{t}\right]^{m-q}\left(\overrightarrow{w_{k}} \cdot \vec{\nabla}\right)^{q} B_{R k}
\end{aligned}
$$

Appendix A.3. Scaling hypothesis

The scaling strategy is justified in section 4.1. The space step $\Delta X$ is the small parameter that is reduced when refining the mesh. The time step is adjusted through diffusive scaling: $\Delta t \propto \Delta X^{2}$. The ratio $(2 \tau-1) / 6 \nu$ is fixed. Thus, we get from Equation 11:

$$
\Delta t=\frac{2 \tau-1}{6 \nu} \Delta X^{2}
$$

This expression is introduced in Equation A.16 


$$
\begin{aligned}
& f_{k}=f_{k}^{e q}+\Delta t \frac{(2 \tau-1)}{2} R_{k} \\
& +\Delta t \tau \sum_{m=1}^{\infty} \sum_{q=0}^{m} \frac{p[\tau, m]}{q !(m-q) !}\left(\frac{2 \tau-1}{6 \nu}\right)^{m-q+1} \Delta X^{2 m-q}\left[\partial_{t}\right]^{m-q}\left(\overrightarrow{w_{k}} \cdot \vec{\nabla}\right)^{q} A_{f k} \\
& +\Delta t \tau \sum_{m=1}^{\infty} \sum_{q=0}^{m} \frac{p[\tau, m]}{q !(m-q) !} 3 \nu\left(\frac{2 \tau-1}{6 \nu}\right)^{m-q+3} \Delta X^{2 m-q+2}\left[\partial_{t}\right]^{m-q}\left(\overrightarrow{w_{k}} \cdot \vec{\nabla}\right)^{q} A_{R k} \\
& +\Delta t \tau \sum_{m=1}^{\infty} \sum_{q=0}^{m} \frac{p[\tau, m]}{q !(m-q) !}\left(\frac{2 \tau-1}{6 \nu}\right)^{m-q} \Delta X^{2 m-q-1}\left[\partial_{t}\right]^{m-q}\left(\overrightarrow{w_{k}} \cdot \vec{\nabla}\right)^{q} B_{f k} \\
& +\Delta t \tau \sum_{m=1}^{\infty} \sum_{q=0}^{m} \frac{p[\tau, m]}{q !(m-q) !} 3 \nu\left(\frac{2 \tau-1}{6 \nu}\right)^{m-q+2} \Delta X^{2 m-q+1}\left[\partial_{t}\right]^{m-q}\left(\overrightarrow{w_{k}} \cdot \vec{\nabla}\right)^{q} B_{R k}
\end{aligned}
$$

Now, there is only one small parameter $(\Delta X)$ in the native equation. The order of magnitude of the different terms will be determined from the power of this parameter.

Appendix A.4. Zero order moment: continuity equation

The continuity equation is obtained by computing the zero order momentum of Equation A.19. We know that $\sum_{k} f_{k}=\sum_{k} f_{k}^{e q}=\rho r$ and $\sum_{k} R_{k}=0$ : all the first line terms are eliminated. The coefficient $\tau \Delta t$ at the begining of the remaining terms is removed:

$$
\begin{aligned}
& 0=\sum_{m=1}^{\infty} \sum_{q=0}^{m} \frac{p[\tau, m]}{q !(m-q) !}\left(\frac{2 \tau-1}{6 \nu}\right)^{m-q+1} \Delta X^{2 m-q}\left[\partial_{t}\right]^{m-q} \sum_{k}\left[\left(\overrightarrow{w_{k}} \cdot \vec{\nabla}\right)^{q} A_{f k}\right] \\
& \sum_{m=1}^{\infty} \sum_{q=0}^{m} \frac{p[\tau, m]}{q !(m-q) !} 3 \nu\left(\frac{2 \tau-1}{6 \nu}\right)^{m-q+3} \Delta X^{2 m-q+2}\left[\partial_{t}\right]^{m-q} \sum_{k}\left[\left(\overrightarrow{w_{k}} \cdot \vec{\nabla}\right)^{q} A_{R k}\right] \\
& \sum_{m=1}^{\infty} \sum_{q=0}^{m} \frac{p[\tau, m]}{q !(m-q) !}\left(\frac{2 \tau-1}{6 \nu}\right)^{m-q} \Delta X^{2 m-q-1}\left[\partial_{t}\right]^{m-q} \sum_{k}\left[\left(\overrightarrow{w_{k}} \cdot \vec{\nabla}\right)^{q} B_{f k}\right] \\
& \sum_{m=1}^{\infty} \sum_{q=0}^{m} \frac{p[\tau, m]}{q !(m-q) !} 3 \nu\left(\frac{2 \tau-1}{6 \nu}\right)^{m-q+2} \Delta X^{2 m-q+1}\left[\partial_{t}\right]^{m-q} \sum_{k}\left[\left(\overrightarrow{w_{k}} \cdot \vec{\nabla}\right)^{q} B_{R k}\right]
\end{aligned}
$$




\begin{tabular}{l|l|l}
\hline & $\Delta X^{0}$ & $\Delta X^{2}$ \\
\hline line 1 $\left(A_{f k}\right)$ & - & $(1,0),(2,2)$ \\
\hline line 2 $\left(A_{R k}\right)$ & - & - \\
\hline line 3 $\left(B_{f k}\right)$ & $(1,1)$ & $(2,1),(3,3)$ \\
\hline line 4 $\left(B_{R k}\right)$ & - & $(1,1)$ \\
\hline
\end{tabular}

Table A.1: Selected combination of $(m, q)$ for the continuity equation

Here, despite the infinite number of terms in the sum, only a few of them have an order of $\Delta X$ lower or equal to 2 . Moreover, for the moments to be non zero, the coefficient $q$ must be even in the two first lines, and odd in the two last lines.

The combinations $(m, q)$ that lead to a $\Delta X$ order lower or equal to 2 , are given in Table A.1 for each line of the former equation. After computing the related moments, we get:

$$
\begin{aligned}
0= & -\partial_{\alpha}\left(\rho r u_{\alpha}\right) \\
& +\frac{(2 \tau-1)^{2}}{12 \nu} \Delta X^{2}\left[-\frac{r}{\nu} \partial_{t} p+2 \partial_{t \alpha}\left(\rho r u_{\alpha}\right)+\partial_{\alpha \beta}\left(r p \delta_{\alpha \beta}+\rho r u_{\alpha} u_{\beta}\right)-\partial_{\alpha}\left(r F_{\alpha}\right)\right] \\
& +\frac{-6 \tau^{2}+6 \tau-1}{6} \Delta X^{2}\left[\partial_{\beta \beta \alpha}\left(\rho r u_{\alpha}\right)\right]+O\left(\Delta X^{4}\right)
\end{aligned}
$$

Appendix A.5. First order moment: momentum equation

The momentum equation is obtained from the first order moments of Equation A.19. For the terms in the first line, we use:

$$
\left\{\begin{array}{l}
\sum_{k} c_{k \alpha} f_{k}=\rho r u_{\alpha}-\frac{\Delta t}{2} r F_{\alpha} \\
\sum_{k} c_{k \alpha} f_{k}^{e q}=\rho r u_{\alpha} \\
\sum_{k} c_{k \alpha} R_{k}=r F_{\alpha}
\end{array}\right.
$$




\begin{tabular}{l|l|l}
\hline & $\Delta X^{0}$ & $\Delta X^{2}$ \\
\hline line 1 $\left(A_{f k}\right)$ & $(1,1)$ & $(2,1),(3,3)$ \\
\hline line 2 $\left(A_{R k}\right)$ & - & $(1,1)$ \\
\hline line 3 $\left(B_{f k}\right)$ & $(1,0),(2,2)$ & $(2,0), \quad(3,2)$, \\
& & $(4,4)$ \\
\hline line 4 $\left(B_{R k}\right)$ & - & $(1,0),(2,2)$ \\
\hline
\end{tabular}

Table A.2: Selected combination of $(m, q)$ for the momentum equation

For the other terms, we use the following development:

$$
\sum_{k} c_{k \alpha}[-]=\frac{\Delta X}{\Delta t} \sum_{k} w_{k \alpha}[-]=\left(\frac{2 \tau-1}{6 \nu}\right)^{-1} \Delta X^{-1} \sum_{k} w_{k \alpha}[-]
$$

The only surviving term in the first line of the first order momentum equation is $\Delta t \tau\left(r F_{\alpha}\right)$. The coefficient $\tau \Delta t$ at the beginning of each line is eliminated. We get:

$$
\begin{aligned}
& 0=r F_{\alpha}+\sum_{m=1}^{\infty} \sum_{q=0}^{m} \frac{p[\tau, m]}{q !(m-q) !}\left(\frac{2 \tau-1}{6 \nu}\right)^{m-q} \Delta X^{2 m-q-1}\left[\partial_{t}\right]^{m-q} \sum_{k}\left[\left(\overrightarrow{w_{k}} \cdot \vec{\nabla}\right)^{q} w_{k \alpha} A_{f k}\right] \\
& \sum_{m=1}^{\infty} \sum_{q=0}^{m} \frac{p[\tau, m]}{q !(m-q) !} 3 \nu\left(\frac{2 \tau-1}{6 \nu}\right)^{m-q+2} \Delta X^{2 m-q+1}\left[\partial_{t}\right]^{m-q} \sum_{k}\left[\left(\overrightarrow{w_{k}} \cdot \vec{\nabla}\right)^{q} w_{k \alpha} A_{R k}\right] \\
& \sum_{m=1}^{\infty} \sum_{q=0}^{m} \frac{p[\tau, m]}{q !(m-q) !}\left(\frac{2 \tau-1}{6 \nu}\right)^{m-q-1} \Delta X^{2 m-q-2}\left[\partial_{t}\right]^{m-q} \sum_{k}\left[\left(\overrightarrow{w_{k}} \cdot \vec{\nabla}\right)^{q} w_{k \alpha} B_{f k}\right] \\
& \sum_{m=1}^{\infty} \sum_{q=0}^{m} \frac{p[\tau, m]}{q !(m-q) !} 3 \nu\left(\frac{2 \tau-1}{6 \nu}\right)^{m-q+1} \Delta X^{2 m-q}\left[\partial_{t}\right]^{m-q} \sum_{k}\left[\left(\overrightarrow{w_{k}} \cdot \vec{\nabla}\right)^{q} w_{k \alpha} B_{R k}\right]
\end{aligned}
$$

The coefficient $q$ must be odd to get non zero moments in the two first lines. It must be even for lines 3 and 4 . The combinations $(m, p)$ for an order of $\Delta X$ equal or lower to 2 are given in Table A.2. The momentum equation at this stage turns into: 


$$
\begin{aligned}
0= & -\partial_{t}\left(\rho r u_{\alpha}\right)-\partial_{\beta}\left(r p \delta_{\alpha \beta}+\rho r u_{\alpha} u_{\beta}\right)+\nu\left[\partial_{\beta \beta}\left(\rho r u_{\alpha}\right)+2 \partial_{\alpha \beta}\left(\rho r u_{\beta}\right)\right]+r F_{\alpha} \\
& +\frac{(2 \tau-1)^{2}}{12 \nu} \Delta X^{2}\left[\partial_{t t}\left(\rho r u_{\alpha}\right)-\partial_{t}\left(r F_{\alpha}\right)+2 \partial_{t \beta}\left(r p \delta_{\alpha \beta}+\rho r u_{\alpha} u_{\beta}\right)-\partial_{\beta}\left(r u_{\alpha} F_{\beta}+r u_{\beta} F_{\alpha}\right)\right] \\
& +\frac{(2 \tau-1)^{2}}{12 \nu} \Delta X^{2} \nu\left[\partial_{\beta \beta}\left(r F_{\alpha}\right)+2 \partial_{\alpha \beta}\left(r F_{\beta}\right)\right] \\
& +\frac{-6 \tau^{2}+6 \tau-1}{6} \Delta X^{2}\left[\partial_{t \beta \beta}\left(\rho r u_{\alpha}\right)+2 \partial_{t \alpha \beta}\left(\rho r u_{\beta}\right)+\partial_{\alpha \beta \beta}\left(r p-\frac{\rho r u_{\gamma \gamma}}{2}\right)+\sum \oplus_{\alpha}\right] \\
& +\frac{12 \tau^{2}-12 \tau+1}{4} \nu \Delta X^{2}\left[\sum \otimes_{\alpha}\right]+O\left(\Delta X^{4}\right)
\end{aligned}
$$

Where:

$$
\begin{aligned}
& \sum \oplus_{\alpha}=\frac{9}{2} \sum_{k} \omega_{k}\left(\overrightarrow{w_{k}} \cdot \vec{\nabla}\right)^{3} w_{k \alpha} \rho r\left(\overrightarrow{w_{k}} \cdot \vec{u}\right) \\
& \sum \otimes_{\alpha}=3 \sum_{k} \omega_{k}\left(\overrightarrow{w_{k}} \cdot \vec{\nabla}\right)^{4} w_{k \alpha} \rho r\left(\overrightarrow{w_{k}} \cdot \vec{u}\right)^{2}
\end{aligned}
$$

Appendix A.6. Extraction of the additional momentum terms

Here come the tricky part of the development. In Equation A.25, only the first line seems to be zero order, the following lines being multiples of $\Delta X^{2}$. However, due to the radius coordinate appearing within space derivatives, and due to the definition of the force term, one additional zero order term arises.

The definition of the force term is:

$$
F_{\alpha}=F_{0 \alpha}+F_{\alpha}^{\prime}=F_{0 \alpha}+\left(\frac{p}{r}-\rho \nu \frac{2 u_{r}}{r^{2}}\right) \delta_{\alpha r}
$$

It is introduced in the element $-\partial_{\beta}\left(r u_{\alpha} F_{\beta}+r u_{\beta} F_{\alpha}\right)$ from Equation A.25 (line 2):

$$
\begin{aligned}
& -\partial_{\beta}\left(r u_{\alpha} F_{\beta}+r u_{\beta} F_{\alpha}\right)=-\partial_{\beta}\left[\delta_{\beta r} u_{\alpha} p+\delta_{\alpha r} u_{\beta} p\right] \\
& \quad+2 \nu \partial_{\beta}\left[\left(u_{\alpha} \delta_{\beta r}+u_{\beta} \delta_{\alpha r}\right) \frac{\rho u_{r}}{r}\right]-\partial_{\beta}\left(r u_{\alpha} F_{0 \beta}+r u_{\beta} F_{0 \alpha}\right)
\end{aligned}
$$


The cross terms $u_{\alpha} p$ and $u_{\beta} p$ are of order -2 . Indeed:

$$
u_{\alpha} p=\frac{12 \nu^{2}}{(2 \tau-1)^{2}} \frac{1}{\Delta X^{2}} \rho u_{\alpha}
$$

They are extracted from the second order elements of Equation A.25, providing additional zero order elements in the momentum equation:

$$
\begin{aligned}
& \frac{(2 \tau-1)^{2}}{12 \nu} \Delta X^{2}\left[-\partial_{\beta}\left\{\frac{12 \nu^{2}}{(2 \tau-1)^{2}} \frac{1}{\Delta X^{2}}\left(\delta_{\alpha r} u_{\beta} \rho+\delta_{\beta r} u_{\alpha} \rho\right)\right\}\right] \\
& =\nu\left[-\partial_{r}\left(\rho u_{\alpha}\right)-\delta_{\alpha r}\left(\partial_{\beta}\left(\rho u_{\beta}\right)\right)\right]=\nu\left[-\partial_{r}\left(\rho u_{\alpha}\right)+\delta_{\alpha r}\left(\frac{-\partial_{\beta}\left(\rho r u_{\beta}\right)}{r}+\frac{\rho u_{r}}{r}\right)\right]
\end{aligned}
$$

The momentum equation then turns into:

$$
\begin{aligned}
0= & -\partial_{t}\left(\rho r u_{\alpha}\right)-\partial_{\beta}\left(r p \delta_{\alpha \beta}+\rho r u_{\alpha} u_{\beta}\right)+ \\
& \nu\left[\partial_{\beta \beta}\left(\rho r u_{\alpha}\right)+2 \partial_{\alpha \beta}\left(\rho r u_{\beta}\right)-\partial_{r}\left(\rho u_{\alpha}\right)+\delta_{\alpha r}\left(\frac{-\partial_{\beta}\left(\rho r u_{\beta}\right)}{r}+\frac{\rho u_{r}}{r}\right)\right]+r F_{\alpha} \\
& +\frac{(2 \tau-1)^{2}}{12 \nu} \Delta X^{2}\left[\partial_{t t}\left(\rho r u_{\alpha}\right)-\partial_{t}\left(r F_{\alpha}\right)+2 \partial_{t \beta}\left(r p \delta_{\alpha \beta}+\rho r u_{\alpha} u_{\beta}\right)-\partial_{\beta}\left(r u_{\alpha} F_{0 \beta}+r u_{\beta} F_{0 \alpha}\right)\right] \\
& +\frac{(2 \tau-1)^{2}}{12 \nu} \Delta X^{2} \nu\left[\partial_{\beta \beta}\left(r F_{\alpha}\right)+2 \partial_{\alpha \beta}\left(r F_{\beta}\right)+2 \partial_{r}\left(\frac{\rho u_{\alpha} u_{r}}{r}\right)+2 \partial_{\beta}\left(\frac{\rho u_{\beta} u_{r}}{r}\right) \delta_{\alpha r}\right] \\
& +\frac{-6 \tau^{2}+6 \tau-1}{6} \Delta X^{2}\left[\partial_{t \beta \beta}\left(\rho r u_{\alpha}\right)+2 \partial_{t \alpha \beta}\left(\rho r u_{\beta}\right)+\partial_{\alpha \beta \beta}\left(r p-\frac{\rho r u_{\gamma \gamma}}{2}\right)+\sum \oplus_{\alpha}\right] \\
& +\frac{12 \tau^{2}-12 \tau+1}{4} \nu X^{2}\left[\sum \otimes_{\alpha}\right]+O\left(\Delta X^{4}\right)
\end{aligned}
$$

\section{Appendix A.\%. Simplifications}

First, we use the zero order terms of Equation A.31 to simplify the continuity equation: 


$$
\begin{aligned}
0= & \left(\text { eq.A.25) }+\frac{(2 \tau-1)^{2}}{12 \nu} \Delta X^{2} \partial_{\alpha}\right. \text { (eq.A.31) } \\
0= & -\partial_{\alpha}\left(\rho r u_{\alpha}\right) \\
& +\frac{(2 \tau-1)^{2}}{12 \nu} \Delta X^{2}\left[-\frac{r}{\nu} \partial_{t}(p)+\partial_{t \alpha}\left(\rho r u_{\alpha}\right)\right] \\
& +\frac{(2 \tau-1)^{2}}{12 \nu} \Delta X^{2} \nu\left[\partial_{\beta \beta \alpha}\left(\rho r u_{\alpha}\right)+2 \partial_{\alpha \alpha \beta}\left(\rho r u_{\beta}\right)-\partial_{r \alpha}\left(\rho u_{\alpha}\right)+\delta_{\alpha r} \partial_{\alpha}\left(\frac{-\partial_{\beta}\left(\rho r u_{\beta}\right)}{r}+\frac{\rho u_{r}}{r}\right)\right] \\
& +\frac{-6 \tau^{2}+6 \tau-1}{6} \Delta X^{2}\left[\partial_{\beta \beta \alpha}\left(\rho r u_{\alpha}\right)\right]+O\left(\Delta X^{4}\right)
\end{aligned}
$$

It is clear that the divergence terms $\partial_{\alpha}\left(\rho r u_{\alpha}\right)$ have an order of magnitude $\Delta X^{2}$. They are removed from the second order elements:

$$
\partial_{\alpha}\left(\rho r u_{\alpha}\right)=\frac{(2 \tau-1)^{2}}{12 \nu} \Delta X^{2}\left[-\frac{r}{\nu} \partial_{t}(p)+\nu \partial_{r}\left(\frac{\rho u_{r}}{r}\right)\right]
$$

The density variations are second order terms:

$$
\partial \rho=\frac{\partial p}{c_{s}^{2}}=\frac{(2 \tau-1)^{2}}{12 \nu} \Delta X^{2} \frac{\partial p}{\nu}
$$

Thus, when extracting the density from the zero order derivative $\partial_{\alpha}\left(\rho r u_{\alpha}\right)$, we must take into account the corresponding second order element in the error terms. However, when we extract the density from derivatives that are already within the second order terms, the equation is not modified (the additional terms would be fourth order). We get the modified continuity equation:

$$
\partial_{\alpha}\left(r u_{\alpha}\right)=\frac{(2 \tau-1)^{2}}{12 \nu} \Delta X^{2}\left[-\frac{r}{\rho \nu}\left(\partial_{t} p+u_{\alpha} \partial_{\alpha} p\right)+\nu \partial_{r}\left(\frac{u_{r}}{r}\right)\right]
$$

For the momentum equation, we begin the simplifications by substituting the divergence terms using Equation A.33: 


$$
\begin{aligned}
0= & -\partial_{t}\left(\rho r u_{\alpha}\right)-\partial_{\beta}\left(r p \delta_{\alpha \beta}+\rho r u_{\alpha} u_{\beta}\right)+\nu\left[\partial_{\beta \beta}\left(\rho r u_{\alpha}\right)-\partial_{r}\left(\rho u_{\alpha}\right)+\delta_{\alpha r} \frac{\rho u_{r}}{r}\right]+r F_{\alpha} \\
& +\frac{(2 \tau-1)^{2}}{12 \nu} \Delta X^{2}\left[\partial_{t t}\left(\rho r u_{\alpha}\right)-\partial_{t}\left(r F_{\alpha}\right)+2 \partial_{t \beta}\left(r p \delta_{\alpha \beta}+\rho r u_{\alpha} u_{\beta}\right)-\partial_{\beta}\left(r u_{\alpha} F_{0 \beta}+r u_{\beta} F_{0 \alpha}\right)\right] \\
& +\frac{(2 \tau-1)^{2}}{12 \nu} \Delta X^{2}\left[\delta_{\alpha r} \partial_{t}(p)-2 \partial_{t \alpha}(r p)\right] \\
& +\frac{(2 \tau-1)^{2}}{12 \nu} \Delta X^{2} \nu\left[\partial_{\beta \beta}\left(r F_{\alpha}\right)+2 \partial_{\alpha \beta}\left(r F_{\beta}\right)+2 \partial_{r}\left(\frac{\rho u_{\alpha} u_{r}}{r}\right)+2 \partial_{\beta}\left(\frac{\rho u_{\beta} u_{r}}{r}\right) \delta_{\alpha r}\right] \\
& +\frac{(2 \tau-1)^{2}}{12 \nu} \Delta X^{2} \nu^{2}\left[2 \partial_{\alpha r}\left(\frac{\rho u_{r}}{r}\right)-\frac{\delta_{\alpha r}}{r} \partial_{r}\left(\frac{\rho u_{r}}{r}\right)\right] \\
& +\frac{-6 \tau^{2}+6 \tau-1}{6} \Delta X^{2}\left[\partial_{t \beta \beta}\left(\rho r u_{\alpha}\right)+2 \partial_{t \alpha \beta}\left(\rho r u_{\beta}\right)+\partial_{\alpha \beta \beta}\left(r p-\frac{\rho r u_{\gamma \gamma}}{2}\right)+\sum \oplus_{\alpha}\right] \\
& +\frac{12 \tau^{2}-12 \tau+1}{4} \nu \Delta X^{2}\left[\sum \otimes_{\alpha}\right]+O\left(\Delta X^{4}\right)
\end{aligned}
$$

The density is taken out of the derivatives:

$$
\begin{aligned}
0= & -\partial_{t}\left(r u_{\alpha}\right)-\partial_{\beta}\left(r u_{\alpha} u_{\beta}\right)-\frac{\partial_{r}(r p)}{\rho}+\nu\left[\partial_{\beta \beta}\left(r u_{\alpha}\right)-\partial_{r}\left(u_{\alpha}\right)+\delta_{\alpha r} \frac{u_{r}}{r}\right]+\frac{r F_{\alpha}}{\rho} \\
& +\frac{(2 \tau-1)^{2}}{12 \nu} \frac{\Delta X^{2}}{\rho \nu}\left[-r u_{\alpha} \partial_{t}(p)-r u_{\alpha} u_{\beta} \partial_{\beta}(p)\right] \\
& +\frac{(2 \tau-1)^{2}}{12 \nu} \Delta X^{2}\left[\partial_{t t}\left(r u_{\alpha}\right)+2 \partial_{t \beta}\left(r u_{\alpha} u_{\beta}\right)\right] \\
& +\frac{(2 \tau-1)^{2}}{12 \nu} \frac{\Delta X^{2}}{\rho}\left[\delta_{\alpha r} \partial_{t}(p)+2 \partial_{\beta}\left(r u_{\alpha}\right) \partial_{\beta}(p)+r u_{\alpha} \partial_{\beta \beta}(p)-u_{\alpha} \partial_{r}(p)\right] \\
& +\frac{(2 \tau-1)^{2}}{12 \nu} \frac{\Delta X^{2}}{\rho}\left[-\partial_{t}\left(r F_{\alpha}\right)-\partial_{\beta}\left(r u_{\alpha} F_{0 \beta}+r u_{\beta} F_{0 \alpha}\right)\right] \\
& +\frac{(2 \tau-1)^{2}}{12 \nu} \Delta X^{2} \nu\left[\frac{\partial_{\beta \beta}\left(r F_{\alpha}\right)+2 \partial_{\alpha \beta}\left(r F_{\beta}\right)}{\rho}+2 \partial_{r}\left(\frac{u_{\alpha} u_{r}}{r}\right)+2 \partial_{\beta}\left(\frac{u_{\beta} u_{r}}{r}\right) \delta_{\alpha r}\right] \\
& +\frac{(2 \tau-1)^{2}}{12 \nu} \Delta X^{2} \nu^{2}\left[2 \partial_{\alpha r}\left(\frac{u_{r}}{r}\right)-\frac{\delta_{\alpha r}}{r} \partial_{r}\left(\frac{u_{r}}{r}\right)\right] \\
& +\frac{-6 \tau^{2}+6 \tau-1}{6} \Delta X^{2}\left[\partial_{t \beta \beta}\left(r u_{\alpha}\right)+2 \partial_{t \alpha \beta}\left(r u_{\beta}\right)+\partial_{\alpha \beta \beta}\left(\frac{r p}{\rho}-\frac{r u_{\gamma \gamma}}{2}\right)+\sum \oplus_{\alpha} / \rho\right] \\
& +\frac{12 \tau^{2}-12 \tau+1}{4} \nu \Delta X^{2}\left[\sum \otimes_{\alpha} / \rho\right]+O\left(\Delta X^{4}\right)
\end{aligned}
$$

We recover on the zero order terms the Navier Stokes momentum after sub- 
stituting the force term $F_{\alpha}$ by its expression in A.27.

\section{Appendix A.8. Comments}

In section Appendix A.6, the additional zero order element was extracted from the force term $F_{\alpha}^{\prime}$. We shall point out that, even if the definition of $F_{\alpha}^{\prime}$ did not contain the pressure $p$, the same additional zero order element would appear from the double time derivative $\partial_{t t}\left(\rho r u_{\alpha}\right)$ in Equation A.25.

The procedure for extracting this term is not so simple. First, we express the zero order part of Equation A.25, by taking into account the additional zero order term through the unknown variable $K_{\alpha}$ :

$$
\begin{aligned}
& 0=-\partial_{t}\left(\rho r u_{\alpha}\right)-\partial_{\beta}\left(r p \delta_{\alpha \beta}+\rho r u_{\alpha} u_{\beta}\right)+ \\
& \nu\left[\partial_{\beta \beta}\left(\rho r u_{\alpha}\right)+2 \partial_{\alpha \beta}\left(\rho r u_{\beta}\right)\right]+r F_{\alpha}+K_{\alpha}+O\left(\Delta X^{2}\right)
\end{aligned}
$$

Then, Equation A.38 is used to substitute the inner time derivative of $\partial_{t}\left(\partial_{t}\left(\rho r u_{\alpha}\right)\right)$. One of the resulting terms is $\partial_{t \beta}\left(\rho r u_{\alpha} u_{\beta}\right)$. We develop this term in the following manner:

$$
\partial_{t \beta}\left(\rho r u_{\alpha} u_{\beta}\right)=\partial_{\beta}\left[u_{\beta} \partial_{t}\left(\rho r u_{\alpha}\right)+u_{\alpha} \partial_{t}\left(\rho r u_{\beta}\right)-u_{\alpha} u_{\beta} \partial_{t}(\rho r)\right]
$$

And finally, we substitute the terms $\partial_{t}\left(\rho r u_{\alpha}\right)$ and $\partial_{t}\left(\rho r u_{\beta}\right)$ using again Equation A.38. The cross terms $p u_{\alpha}$ and $p u_{\beta}$ appear naturally. In our particular case, those cross terms are cancelled by the elements $u_{\alpha} F_{\alpha}{ }^{\prime}$ and $u_{\beta} F_{\beta}{ }^{\prime}$ which also contain the pressure.

[1] U. Frisch, B. Hasslacher, and Y. Pomeau. Lattice-gas automata for the navier-stokes equation. Phys. Rev. Lett., 56:1505-1508, Apr 1986.

[2] Guy R. McNamara and Gianluigi Zanetti. Use of the boltzmann equation to simulate lattice-gas automata. Phys. Rev. Lett., 61:2332-2335, Nov 1988. 
[3] Xiaoyi He and Li-Shi Luo. Theory of the lattice boltzmann method: From the boltzmann equation to the lattice boltzmann equation. Phys. Rev. E, 56:6811-6817, Dec 1997.

[4] Y. H. Qian, D. D'Humière, and P. Lallemand. Lattice bgk models for navier-stokes equation. Europhysics Letters, 17:479-484, 1992.

[5] Zhenhua Chai and Baochang Shi. A novel lattice boltzmann model for the poisson equation. Applied Mathematical Modelling, 32(10):2050 - 2058, 2008.

[6] Subhash C. Mishra, Anjaneyulu Lankadasu, and Kamen N. Beronov. Application of the lattice boltzmann method for solving the energy equation of a 2-d transient conduction-radiation problem. International Journal of Heat and Mass Transfer, 48(17):3648 - 3659, 2005.

[7] Fuzhang Zhao. Optimal relaxation collisions for lattice boltzmann methods. Computers \& Mathematics with Applications, 65(2):172 - 185, 2013. Special Issue on Mesoscopic Methods in Engineering and Science (ICMMES2010, Edmonton, Canada).

[8] David J Holdych, David R Noble, John G Georgiadis, and Richard O Buckius. Truncation error analysis of lattice boltzmann methods. Journal of Computational Physics, 193(2):595 - 619, 2004.

[9] Michael Junk. A finite difference interpretation of the lattice boltzmann method. Numerical Methods for Partial Differential Equations, 17(4):383402, 2001.

[10] Irina Ginzburg. Truncation errors, exact and heuristic stability analysis of two-relaxation-times lattice boltzmann schemes for anisotropic advectiondiffusion equation. International Journal for Multiscale Computational Engineering, 11:1439-1502, 2012. 
[11] Zhaoli Guo, Haifeng Han, Baochang Shi, and Chuguang Zheng. Theory of the lattice boltzmann equation: Lattice boltzmann model for axisymmetric flows. Phys. Rev. E, 79:046708, Apr 2009.

[12] I. Halliday, L. A. Hammond, C. M. Care, K. Good, and A. Stevens. Lattice boltzmann equation hydrodynamics. Phys. Rev. E, 64:011208, Jun 2001.

[13] T. Reis and T. N. Phillips. Modified lattice boltzmann model for axisymmetric flows. Phys. Rev. E, 75:056703, May 2007.

[14] T. Reis and T. N. Phillips. Erratum: Modified lattice boltzmann model for axisymmetric flows [phys. rev. e 75, 056703 (2007)]. Phys. Rev. E, 76:059902, Nov 2007.

[15] Sheng Chen, Jonas Tölke, Sebastian Geller, and Manfred Krafczyk. Lattice boltzmann model for incompressible axisymmetric flows. Phys. Rev. E, 78:046703, Oct 2008 .

[16] Sheng Chen, Jonas Tölke, and Manfred Krafczyk. Simulation of buoyancydriven flows in a vertical cylinder using a simple lattice boltzmann model. Phys. Rev. E, 79:016704, Jan 2009.

[17] Sheng Chen, Jonas Tölke, and Manfred Krafczyk. Numerical simulation of fluid flow and heat transfer inside a rotating disk-cylinder configuration by a lattice boltzmann model. Phys. Rev. E, 80:016702, Jul 2009.

[18] Sheng Chen, Jonas Tölke, and Manfred Krafczyk. Simple lattice boltzmann subgrid-scale model for convectional flows with high rayleigh numbers within an enclosed circular annular cavity. Phys. Rev. E, 80:026702, Aug 2009.

[19] Jian Guo Zhou. Axisymmetric lattice boltzmann method. Phys. Rev. E, 78:036701, Sep 2008.

[20] Jian Guo Zhou. Axisymmetric lattice boltzmann method revised. Phys. Rev. E, 84:036704, Sep 2011. 
[21] Diethard Bergers. Kinetic model solution for axisymmetric flow by the method of discrete ordinates. Journal of Computational Physics, 57(2):285 $-302,1985$.

[22] Lin Zheng, Baochang Shi, Zhaoli Guo, and Chuguang Zheng. Lattice boltzmann equation for axisymmetric thermal flows. Computers \& Fluids, 39(6):945 - 952, 2010.

[23] Liang Wang, Zhaoli Guo, and Chuguang Zheng. Multi-relaxation-time lattice boltzmann model for axisymmetric flows. Computers \& Fluids, 39(9):1542 - 1548, 2010.

[24] Xiaoyi He, Xiaowen Shan, and Gary D. Doolen. Discrete boltzmann equation model for nonideal gases. Phys. Rev. E, 57:R13-R16, Jan 1998.

[25] Zhaoli Guo, Chuguang Zheng, and Baochang Shi. Discrete lattice effects on the forcing term in the lattice boltzmann method. Phys. Rev. E, 65:046308, Apr 2002.

[26] Pietro Asinari, Taku Ohwada, Eliodoro Chiavazzo, and Antonio F. Di Rienzo. Link-wise artificial compressibility method. J. Comput. Phys., 231(15):5109-5143, June 2012.

[27] Paul J. Dellar. An interpretation and derivation of the lattice boltzmann method using strang splitting. Computers \& Mathematics with Applications, 65(2):129 - 141, 2013.

[28] David R. Noble, Shiyi Chen, John G. Georgiadis, and Richard O. Buckius. A consistent hydrodynamic boundary condition for the lattice boltzmann method. Physics of Fluids (1994-present), 7(1):203-209, 1995.

[29] Martin Hecht and Jens Harting. Implementation of on-site velocity boundary conditions for d3q19 lattice boltzmann simulations. Journal of Statistical Mechanics: Theory and Experiment, 2010(01):P01018, 2010. 
[30] John C. Tannehill, Dale Dale Arden Anderson, and Richard H. Pletcher. Computational Fluid Mechanics and Heat Transfer, Second Edition. Taylor \& Francis, 1997.

[31] A. Lemembre and J.-P. Petit. Laminar natural convection in a laterally heated and upper cooled vertical cylindrical enclosure. International Journal of Heat and Mass Transfer, 41(16):2437 - 2454, 1998. 